\title{
A locally conservative variational multiscale method for the simulation of porous media flow with multiscale source terms
}

\author{
by \\ Francois-Xavier Dub
}

Submitted to the Department of Mechanical Engineering in partial fulfillment of the requirements for the degree of

Master of Science in Mechanical Engineering

at the
MASSACHUSETTS INSTITUTE OF TECHNOLOGY [June 2008]
May 2008

(c) Massachusetts Institute of Technology 2008. All rights reserved.

Author ......................

Department of Mechanical Engineering

Certified by 大 ...May 9, 2008
/ - Ruben Juanes
Assistant Professor, Civil and Environmental Engineering Thesis Supervisor

Certified by Anthony T. Patera
Professor, Mechanical Engineering
Department Internal Advisor

Accepted by .........

Lallit Anand

Chairman, Department Committee on Graduate Students OF TEOHNOLOGY

JUL 292008

I IODADIEA 


\title{
A locally conservative variational multiscale method for the simulation of porous media flow with multiscale source terms
}

by

\author{
Francois-Xavier Dub
}

\author{
Submitted to the Department of Mechanical Engineering \\ on May 9,2008, in partial fulfillment of the \\ requirements for the degree of \\ Master of Science in Mechanical Engineering
}

\begin{abstract}
Multiscale phenomena are ubiquitous to flow and transport in porous media. They manifest themselves through at least the following three facets: (1) effective parameters in the governing equations are scale dependent; (2) some features of the flow (especially sharp fronts and boundary layers) cannot be resolved on practical computational grids; and (3) dominant physical processes may be different at different scales. Numerical methods should therefore reflect the multiscale character of the solution. We concentrate on the development of simulation techniques that account for the heterogeneity present in realistic reservoirs, and have the ability to solve for coupled pressure-saturation problems (on coarse grids). We present a variational multiscale mixed finite element method for the solution of Darcy flow in porous media, in which both the permeability field and the source term display a multiscale character. The formulation is based on a multiscale split of the solution into coarse and subgrid scales. This decomposition is invoked in a variational setting that leads to a rigorous definition of a (global) coarse problem and a set of (local) subgrid problems. One of the key issues for the success of the method is the proper definition of the boundary conditions for the localization of the subgrid problems. We identify a weak compatibility condition that allows for subgrid communication across element interfaces, something that turns out to be essential for obtaining high-quality solutions. We also remove the singularities due to concentrated sources from the coarse-scale problem by introducing additional multiscale basis functions, based on a decomposition of fine-scale source terms into coarse and deviatoric components. The method is locally conservative and employs a low-order approximation of pressure and velocity at both scales. We illustrate the performance of the method on several synthetic cases, and conclude that the method is able to capture the global and local flow patterns accurately.
\end{abstract}

Thesis Supervisor: Ruben Juanes

Title: Assistant Professor, Civil and Environmental Engineering 
Department Internal Advisor: Anthony T. Patera

Title: Professor, Mechanical Engineering 


\section{Acknowledgments}

I consider myself very lucky my thesis research was supervised by Ruben Juanes. He has continually challenged my ideas and given me able guidance, while providing a friendly work atmosphere. I am grateful to him for his patience, encouragement and for understanding my decision not to pursue a $\mathrm{PhD}$.

I am very thankful to my labmates at Parsons: Mojtaba Bateni, Homero Flores, Rebecca Gianotti, Antone Jain, Mathilde Lerminiaux, Christopher MacMinn, Gajan Sivandran, Laure-Anne Ventouras... for making the lab such a lively place.

I am so thankful to my family for being so supportive during this time. Thank you to my grand-parents, my parents, my brothers and my sister.

Thank you to all my friends at MIT or outside MIT who made my 2 years in Boston a memorable moment in my life: Blandine Antoine, Christophe Antoine, Julien Beccherle, Catherine Bolliet, Jeff Chambers, Noemie Chocat, Stephane Chong, Tuly Gonzalez, Laurent Guerin, David Hemous, Romain Lacombe, Francois Le Floch, Crystal Joyce, Coppelia Marincovic, Thibaud Normand, Thibault Peyronel, Samuel Ronsin, Guillaume de Roo... and thank you especially to Amandine Darras, Edouard Gueyffier and Anne-Gabrielle Laboureau (in New-York) ; Marie Naveau, Louis de Rouge (in Paris) ; Suzanne de Bourgoing, Isabelle Delefortrie and Gregoire Villeroy (in Boston). 


\section{Contents}

1 Mathematical formulation $\quad 17$

1.1 Governing equations . . . . . . . . . . . . . . 17

1.2 Mixed variational formulation . . . . . . . . . . . . 18

1.3 The mixed finite element method . . . . . . . . . . . . . 19

2 The variational multiscale method 21

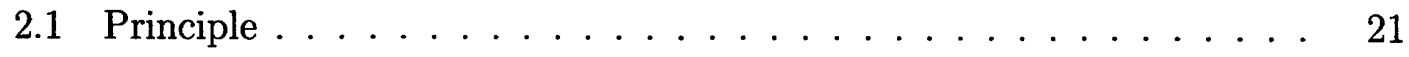

2.2 Choice of finite element spaces . . . . . . . . . . . . . 23

2.3 Localization of the subgrid problem . . . . . . . . . . . 25

2.4 Boundary conditions for the local subgrid problems . . . . . . . . 27

2.4.1 No subgrid communication . . . . . . . . . . . 27

2.4.2 Proposed subgrid communication . . . . . . . . . . 28

2.5 The coarse-scale problem revisited . . . . . . . . . . . . . . . 34

2.6 A multiscale method with a coarse pressure approximation . . . . . 35

2.6 .1 Implementation . . . . . . . . . . . . 36

3 Multiscale source terms $\quad 39$

3.1 Principle .......................... 40

3.2 The local well problem . . . . . . . . . . . . . . 41

3.3 The coarse-scale problem revisited . . . . . . . . . . . . 43

4 Numerical simulations $\quad \mathbf{4 5}$

4.1 Small channelized system . . . . . . . . . . . . . . . 46 
4.2 Quarter five-spot simulations . . . . . . . . . . . . . . . . 47

4.2.1 Isotropic correlation structure . . . . . . . . . . . . . . 48

4.2.2 System with vertical channels ... . . . . . . . . . 50

4.2.3 System with diagonal channels . . . . . . . . . . . . . 53

4.3 Study of anisotropy . . . . . . . . . . . . . . 55

4.4 Simulations in realistic $2 \mathrm{D}$ reservoirs f . . . . . . . . . 59

4.4.1 Highly heterogeneous, smoothly-varying permeability . . . . . 59

4.4.2 Highly heterogeneous, channelized permeability . . . . . . 62

4.4 .3 All layers ....................... 67 


\section{List of Figures}

2-1 Diagram illustrating the local flow problem defining the multiscale basis functions. . . . . . . . . . . . . . . . . . . 30

3-1 Diagram illustrating the local flow problem defining the well basis func-

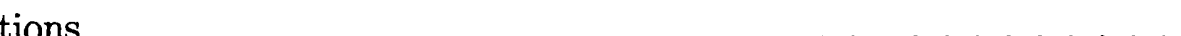

4-1 Small channelized system. Fine-scale finite volume solution. . . . . .

4-2 Small channelized system. Variational multiscale solution on a $2 \times 2$ coarse grid. . . . . . . . . . . . . . . . 47

4-3 Schematic of the quarter five-spot problem. . . . . . . . . . . . 48

4-4 Log-permeability field for the isotropic correlation structure. . . . . . 49

4-5 Isotropic correlation structure. Finite volume solution on the fine $64 \times$

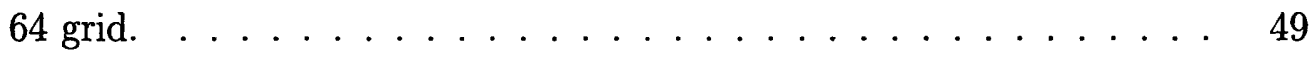

4-6 Isotropic correlation structure. Variational multiscale solution on a $4 \times 4$ grid $(16 \times 16$ upgridding $)$ with well length scale $N_{l}=1 / 4 \ldots$

4-7 Velocity (left) and pressure (right) errors for the isotropic correlation scenario. . . . . . . . . . . . . . . . . . 50

4-8 Log-permeability field for the structure dominated by vertical channels. 51

4-9 Isotropic correlation structure. Finite volume solution on the fine $64 \times$ 64 grid. . . . . . . . . . . . . . . . . 51

4-10 Isotropic correlation structure. Variational multiscale solution on a $4 \times 4$ grid $(16 \times 16$ upgridding $)$ with well length scale $N_{l}=1 / 4 \ldots \ldots$

4-11 Velocity (left) and pressure (right) errors for the scenario with vertical channels. 
4-12 Log-permeability field for the structure dominated by diagonal channels. 53

4-13 Structure dominated by diagonal channels. Finite volume solution on the fine $64 \times 64$ grid. . . . . . . . . . . . . . . . 54

4-14 Structure dominated by diagonal channels. Variational multiscale solution on a $4 \times 4$ grid $\left(16 \times 16\right.$ upgridding) with well length scale $N_{l}=1 / 4.54$

4-15 Velocity (left) and pressure (right) errors for the scenario with diagonal channels. . . . . . . . . . . . . . . . . 54

4-16 Log-permeability $\left(k_{x x}\right)$ field for the study of anisotropy. . . . . . . . 56

4-17 Pressure field for the anisotropic permeability field $(\alpha=0.01)$. Left: fine-scale finite volume solution. Right: variational multiscale solution on a $8 \times 8$ grid $(8 \times 8$ upgridding $) . \ldots \ldots \ldots 7$

4-18 Pressure field for the anisotropic permeability field $(\alpha=0.1)$. Left: fine-scale finite volume solution. Right: variational multiscale solution on a $8 \times 8$ grid $(8 \times 8$ upgridding $) . \ldots \ldots \ldots$

4-19 Pressure field for the isotropic permeability field $(\alpha=1)$. Left: finescale finite volume solution. Right: variational multiscale solution on a $8 \times 8$ grid $(8 \times 8$ upgridding $) . \ldots \ldots \ldots$

4-20 Velocity (left) and pressure (right) errors for the anisotropic permeability field. . . . . . . . . . . . . . . .

4-21 Smooth permeability field. Finite volume solution on the fine $60 \times$

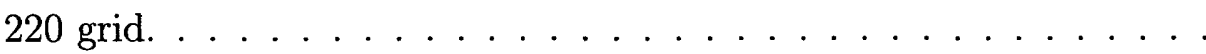

4-22 Smooth permeability field. Variational multiscale solution on a $6 \times$ 22 grid $\left(10 \times 10\right.$ upgridding) with well length scale $N_{l}=1 / 4 \ldots \ldots$

4-23 Smooth permeability field. Velocity and pressure errors for different coarse discretizations. . . . . . . . . . . . . . . .

4-24 Channelized permeability field. Finite volume solution on the fine $60 \times$ 220 grid.

4-25 Channelized permeability field. Variational multiscale solution on a $6 \times 22 \operatorname{grid}(10 \times 10$ upgridding $)$ with well length scale $N_{l}=1 / 4 \ldots$. 
4-26 Channelized permeability field. Velocity and pressure errors for different coarse discretizations. . . . . . . . . . . . . . . 65

4-27 Concentration maps at breakthrough with fluxes obtained by (a) Finite volume method on the fine $60 \times 220$ grid; (b) VMS on a $6 \times 22$ grid; (b) VMS on a $30 \times 22$ grid; (b) VMS on a $30 \times 55$ grid. . . . . . . . 66

4-28 Breakthrough curves of the passive tracer at each of the production wells, computed using fine-scale fluxes from different coarse-grid discretizations. Clockwise, from top-left: Top-left well; Top-right well; Bottom-right well; Bottom-left well. . . . . . . . . . . . . 67

4-29 All layers of the 10th SPE comparative solution project. Velocity error. 68 4-30 All layers of the 10th SPE comparative solution project. Concentration error. . . . . . . . . . . . . . . . . 70

4-31 Comparison of $\Delta_{\mathrm{BHP}}$ between the reference finite volume solution and the variational multiscale solution with 100 -fold upgridding for all layers of the 10th SPE comparative solution project. . . . . . . . . . 


\section{Introduction}

The equations governing the flow of fluids in the subsurface (oil and gas reservoirs, confined aquifers, vadose zone, etc.) can, in many cases, be formulated in terms of a single "pressure" equation (of elliptic character) describing an overall mass balance, and "component" equations (of hyperbolic character) governing the differential displacement of each component [8].

In this work, we concentrate on the numerical solution of a model pressure equation:

$$
\nabla \cdot(-\mathbf{k} \nabla p)=f
$$

where the coefficient tensor $\mathbf{k}$ is discontinuous, highly variable, and may present short and long correlation lengths. Moreover, the source/sink term $f$ displays a multiscale character also. In practical scenarios, flow is driven by injection and production wells. Because wells are features that are much smaller than the grid size, they must be understood as concentrated (point or line) sources.

A number of approaches are currently being investigated for the numerical simulation of porous media flow with rough permeability fields. We can identify at least two main tracks: multiscale finite element or finite volume methods, and variational multiscale methods.

The multiscale finite element method was originally proposed in [20] for the solution of elliptic equations with rapidly oscillating coefficients. The main idea is to construct finite element basis functions which themselves are solutions to the elliptic operator inside each element and, therefore, capture small scale information [11, 10]. The method was analyzed in a series of subsequent papers (see, e.g. $[21,19]$ ). A 
mixed finite element version that guarantees local mass conservation at the element level was proposed in [16]. This work was recently extended in a number of important ways by the Norwegian school [1,2]. Inspired in the multiscale finite element method, a multiscale finite volume method was proposed in [24]. This method also preserves mass conservation at the coarse and fine scales (see also [25, 29]).

The variational multiscale (VMS) method was originally proposed by Hughes ([22, 23]) as an overarching framework for the solution of partial differential equations that exhibit multiscale phenomena (either due to small-scale heterogeneity or sharp features that cannot be captured on a coarse grid). The essence of the method is to perform a multiscale split of the solution into a coarse-scale part (that can be approximated on a coarse grid) and a subscale component. The multiscale split is invoked in a variational setting, which leads to a rigorous definition of a coarse-scale problem and a subgrid-scale problem. While an approximation (localization) of the subgrid problem is typically necessary, the framework offers a rigorous formulation for incorporating subgrid effects in the coarse scale equations. A mixed variant of the method, coined "numerical subgrid upscaling" was developed independently by Arbogast $[3,4,7,5]$.

Links between the mixed version of the variational multiscale approach and the mixed multiscale finite element method have been pointed out recently [6]. A thorough comparison of the different multiscale methods (as well as interesting extensions) has recently been presented in [28].

We adopt the variational multiscale framework to develop a locally conservative multiscale method and we generalize the formulation presented in [26] to account for the presence of source terms with a multiscale character [27]. The main contributions are:

1. We propose an enhanced localization assumption needed to define the local subgrid problems, which allows for subgrid communication across element interfaces and boundaries.

2. We introduce multiscale "well" basis functions, based on a decomposition of 
fine-scale source terms into coarse and deviatoric components.

One of the main features of the proposed method is the relaxed localization assumption with respect to the numerical subgrid upscaling method [4], which will be discussed in Section 2.4.2. The proposed approach to handle concentrated sources is fundamentally different from the one proposed in [35], as is shown in Section 3.2.

In Chapter 1 we introduce the governing equations and the mixed finite element approximation of the global fine-scale problem. The variational multiscale mixed finite element method is developed in Chapter 2, with special emphasis on the enhanced localization assumption we propose. In Chapter 3 we extend the method to incorporate multiscale source terms. In Chapter 4 we illustrate the performance of the proposed method on a number of challenging simulations. We finally draw the main conclusions of the investigation. 


\section{Chapter 1}

\section{Mathematical formulation}

\subsection{Governing equations}

We shall use the following model pressure equation:

$$
\nabla \cdot \boldsymbol{u}=f \quad \text { in } \Omega
$$

where $f$ is the source term, which may be highly variable and display a multiscale character, and $\boldsymbol{u}$ is the total velocity given by Darcy's law:

$$
\boldsymbol{u}=-\mathbf{k} \nabla p
$$

The symbol $\mathbf{k}$ is the permeability tensor and $p$ is the pressure. In a more general setting (multiphase flow problems including gravity effects) $\mathbf{k}$ is the total mobility tensor, and $p$ is the flow potential. The permeability tensor is symmetric and positive definite. The components of $\mathbf{k}$ are assumed to be bounded, but they may be highly discontinuous and display large anisotropy ratios. In this work we shall assume that $\mathbf{k}$ is a diagonal tensor. The pressure equation is supplemented with the following 
boundary conditions:

$$
\begin{aligned}
p=\bar{p} & \text { on } \Gamma_{p}, \\
\boldsymbol{u} \cdot \boldsymbol{n}=\bar{u} & \text { on } \Gamma_{u},
\end{aligned}
$$

where $\Gamma_{p} \cap \Gamma_{u}=\emptyset, \Gamma_{p} \cup \Gamma_{u}=\partial \Omega$, and $\boldsymbol{n}$ is the outward unit normal to the boundary. For expositional simplicity, and without loss of generality (see, e.g. Section IV.1 of [14]), we may take a homogeneous Neumann boundary condition:

$$
\bar{u}=0 \quad \text { on } \Gamma_{u} .
$$

\subsection{Mixed variational formulation}

We write Equations (1.1)-(1.2) in the following form:

$$
\begin{aligned}
\mathbf{k}^{-1} \boldsymbol{u}+\nabla p & =0 \\
\nabla \cdot \boldsymbol{u} & =f .
\end{aligned}
$$

We introduce the following functional spaces:

$$
W \equiv L^{2}(\Omega)=\left\{q: \int_{\Omega}|q|^{2} \mathrm{~d} \Omega=\|q\|_{L^{2}(\Omega)}^{2}<+\infty\right\},
$$

with inner product

$$
(q, p) \equiv(q, p)_{L^{2}(\Omega)}:=\int_{\Omega} q p \mathrm{~d} \Omega, \quad q, p \in L^{2}(\Omega)
$$

and

$$
H(\operatorname{div}, \Omega)=\left\{\boldsymbol{v}: \boldsymbol{v} \in\left(L^{2}(\Omega)\right)^{2}, \nabla \cdot \boldsymbol{v} \in L^{2}(\Omega)\right\}
$$

with inner product

$$
(\boldsymbol{v}, \boldsymbol{u}) \equiv(\boldsymbol{v}, \boldsymbol{u})_{H(\operatorname{div}, \Omega)}:=\int_{\Omega} \boldsymbol{v} \cdot \boldsymbol{u} \mathrm{d} \Omega, \quad \boldsymbol{v}, \boldsymbol{u} \in H(\operatorname{div}, \Omega) .
$$


The space $L^{2}(\Omega)$ is the usual Sobolev space of square integrable functions in $\Omega$. The space $H(\operatorname{div}, \Omega)$ is defined such that a vector $\boldsymbol{v}$ belonging to this space admit a welldefined normal trace on $\partial \Omega$ [14, Section III.1.1]:

$$
\bar{v} \equiv \boldsymbol{v} \cdot \boldsymbol{n} \in H^{-1 / 2}(\partial \Omega)
$$

We will also make use of the following space:

$$
V \equiv H_{0, u}(\operatorname{div}, \Omega)=\left\{\boldsymbol{v}: \boldsymbol{v} \in H(\operatorname{div}, \Omega), \boldsymbol{v} \cdot \boldsymbol{n}=0 \text { on } \Gamma_{u}\right\}
$$

of functions in $H(\operatorname{div}, \Omega)$ with null normal trace on the Neumann boundary $\Gamma_{u}$. Denoting by $H^{1 / 2}(\Gamma)$ the dual space of $H^{-1 / 2}(\Gamma)$ for $\Gamma \subset \partial \Omega$, we also define the duality product:

$$
\langle\bar{u}, \bar{p}\rangle_{\Gamma}:=\int_{\Gamma} \bar{u} \bar{p} \mathrm{~d} \Gamma, \quad \bar{u} \in H^{-1 / 2}(\Gamma), \bar{p} \in H^{1 / 2}(\Gamma) .
$$

Making use of the functional spaces defined above, we can express the problem given by Equations (1.6)-(1.7) with boundary conditions (1.3)-(1.5) in mixed variational form: Find $(\boldsymbol{u}, p) \in V \times W$ such that

$$
\begin{array}{rlrl}
\left(\boldsymbol{v}, \mathbf{k}^{-1} \boldsymbol{u}\right)-(\nabla \cdot \boldsymbol{v}, p) & =-\langle\boldsymbol{v} \cdot \boldsymbol{n}, \bar{p}\rangle_{\Gamma_{p}} & & \forall \boldsymbol{v} \in V \\
(w, \nabla \cdot \boldsymbol{u}) & =(w, f) & \forall w \in W .
\end{array}
$$

It is well known that this problem has a unique solution [14].

\subsection{The mixed finite element method}

The mixed variational formulation provides the basis for the mixed finite element method. Let $V_{h} \subset V$, and $W_{h} \subset W$ be finite dimensional subspaces of the corresponding continuum spaces, the mixed finite element approximation of (1.15)-(1.16) 
reads: Find $\left(\boldsymbol{u}_{h}, p_{h}\right) \in V_{h} \times W_{h}$ such that

$$
\begin{aligned}
\left(\boldsymbol{v}_{h}, \mathbf{k}^{-1} \boldsymbol{u}_{h}\right)-\left(\nabla \cdot \boldsymbol{v}_{h}, p_{h}\right) & =-\left\langle\boldsymbol{v}_{h} \cdot \boldsymbol{n}, \bar{p}\right\rangle_{\Gamma_{p}} & & \forall \boldsymbol{v}_{h} \in V_{h} \\
\left(w_{h}, \nabla \cdot \boldsymbol{u}_{h}\right) & =\left(w_{h}, f\right) & & \forall w_{h} \in W_{h}
\end{aligned}
$$

The spaces $V_{h}$ and $W_{h}$ cannot be chosen independently; they must satisfy a standard coercivity condition and the discrete inf-sup condition $[9,12]$. The numerical solution of (1.17)-(1.18) invariably involves a partition $\mathcal{T}_{h}$ of the domain $\Omega$ into nonoverlapping elements $e_{i}, \mathcal{T}_{h}=\bigcup_{i=1}^{N_{h}} e_{i}$, where $N_{h}$ is the number of elements of the grid. We also define the skeleton of the partition, $\mathcal{S}_{h}=\bigcup_{a=1}^{M_{h}} \gamma_{a}$, where $M_{h}$ is the number of element faces denoted by $\gamma_{a}$. We shall understand the partition $\mathcal{T}_{h}$ as the fine grid, on which the permeability $\mathbf{k}$ is defined. For definiteness, we shall use a partition into rectangular elements, and associate the fine-scale velocity space with the lowest-order Raviart-Thomas space, $R T_{0}\left(\mathcal{T}_{h}\right)[32]$ :

$$
V_{h}=\left\{\boldsymbol{v}_{h}: \boldsymbol{v}_{h}=\sum_{a=1}^{M_{h}} \boldsymbol{N}_{a}^{h} \mathrm{v}_{a}, \quad \mathrm{v}_{b}=0 \forall \gamma_{b} \in \Gamma_{u}\right\}
$$

where $\boldsymbol{N}_{a}^{h}$ is the $R T_{0}$ basis function associated with face $\gamma_{a}$, and $\mathrm{v}_{a}$ is the corresponding degree of freedom (the integrated flux through face $\gamma_{a}$ ). The corresponding pressure approximation is piecewise constant on the fine mesh, $P_{0}\left(\mathcal{T}_{h}\right)$ :

$$
W_{h}=\left\{w_{h}: w_{h}=\sum_{i=1}^{N_{h}} \chi_{i}^{h} \mathbf{w}_{i}\right\}
$$

where $\chi_{i}^{h}$ is the characteristic function for element $i$ (equal to one at element $e_{i}$, zero at all other elements), and $w_{i}$ is the corresponding degree of freedom (the average pressure at element $e_{i}$ ).

Other choices of velocity and pressure spaces are of course possible [14]. The $R T_{0}$ space is, however, the simplest. Moreover, it can be shown that for diagonal permeability tensor, and under appropriate numerical quadrature, this method reduces to the traditional finite difference method $[33,34]$. 


\section{Chapter 2}

\section{The variational multiscale method}

\subsection{Principle}

The essence of the variational multiscale method $[22,23]$ is to perform a multiscale split of the solution into a coarse-scale part (that can be approximated on a coarse grid) and a subscale component. The multiscale split is invoked in a variational setting, which leads to a rigorous definition of a coarse-scale problem and a subgrid-scale problem. By virtue of this decomposition, we acknowledge that the fine-scale details of the solution cannot be captured on a coarse grid. As it turns out, inaccuracies at the subgrid level may resonate, and produce a numerical solution that is globally polluted with errors if one does not model subgrid effects correctly. We show that high-fidelity fine-scale solutions can be obtained by properly accounting for subgridscale heterogeneity.

Although the variational multiscale formalism is general and can be applied to the continuum problem $[22,5,26]$, here we restrict our attention to the discrete finescale problem. Consider a coarse partition of the domain $\mathcal{T}_{H}=\bigcup_{i=1}^{N_{H}} E_{i}$ and the associated skeleton $\mathcal{S}_{H}=\bigcup_{a=1}^{M_{H}} \Gamma_{a}$ on which a coarse-scale discretization is defined, 
and the decomposition of the fine-scale solution:

$$
\begin{aligned}
& \boldsymbol{u}_{h}=\boldsymbol{u}_{H}+\tilde{\boldsymbol{u}} \\
& p_{h}=p_{H}+\tilde{p} .
\end{aligned}
$$

This decomposition is unique if we can express the original fine-scale solution space $V_{h} \times W_{h}$ as the direct sum of two spaces, with:

$$
\begin{gathered}
V_{h}=V_{H} \oplus \tilde{V}, \\
W_{h}=W_{H} \oplus \tilde{W},
\end{gathered}
$$

where $V_{H} \times W_{H}$ is the space of coarse scales, and $\tilde{V} \times \tilde{W}$ is the space of subgrid scales. This decomposition allows one to split the fine-scale problem (1.17)-(1.18) into a coarse-scale problem and a subscale problem. Testing against coarse-scale test functions we obtain the coarse-scale problem: Find $\left(\boldsymbol{u}_{H}, p_{H}\right) \in V_{H} \times W_{H}$ such that

$$
\begin{aligned}
\left(\boldsymbol{v}_{H}, \mathbf{k}^{-1} \boldsymbol{u}_{H}\right)+\left(\boldsymbol{v}_{H}, \mathbf{k}^{-1} \tilde{\boldsymbol{u}}\right)-\left(\nabla \cdot \boldsymbol{v}_{H}, p_{H}\right)- & \left(\nabla \cdot \boldsymbol{v}_{H}, \tilde{p}\right) \\
& =-\left\langle\boldsymbol{v}_{H} \cdot \boldsymbol{n}, \bar{p}\right\rangle_{\Gamma_{p}}, \\
\left(w_{H}, \nabla \cdot \boldsymbol{u}_{H}\right)+\left(w_{H}, \nabla \cdot \tilde{\boldsymbol{u}}\right)= & \left(w_{H}, f\right)
\end{aligned}
$$

for all $\boldsymbol{v}_{H} \in V_{H}$ and $w_{H} \in W_{H}$. Testing against the subscale test functions, and expressing the inner product as sums over coarse elements $E_{i}, i=1, \ldots, N_{H}$, we arrive at the subscale problem: Find $(\tilde{\boldsymbol{u}}, \tilde{p}) \in \tilde{V} \times \tilde{W}$ such that

$$
\begin{array}{r}
\sum_{i=1}^{N_{H}}\left[\left(\tilde{\boldsymbol{v}}, \mathbf{k}^{-1} \boldsymbol{u}_{H}\right)_{E_{i}}+\left(\tilde{\boldsymbol{v}}, \mathbf{k}^{-1} \tilde{\boldsymbol{u}}\right)_{E_{i}}-\left(\nabla \cdot \tilde{\boldsymbol{v}}, p_{H}\right)_{E_{i}}-(\nabla \cdot \tilde{\boldsymbol{v}}, \tilde{p})_{E_{i}}\right] \\
=-\sum_{i=1}^{N_{H}}\langle\tilde{\boldsymbol{v}} \cdot \boldsymbol{n}, \bar{p}\rangle_{\Gamma_{p} \cap \partial E_{i}} \\
\sum_{i=1}^{N_{H}}\left[\left(\tilde{w}, \nabla \cdot \boldsymbol{u}_{H}\right)_{E_{i}}\right]+\sum_{i=1}^{N_{H}}\left[(\tilde{w}, \nabla \cdot \tilde{\boldsymbol{u}})_{E_{i}}\right]=\sum_{i=1}^{N_{H}}(\tilde{w}, f)_{E_{i}} .
\end{array}
$$

for all $\tilde{\boldsymbol{v}} \in \tilde{V}$ and $\tilde{w} \in \tilde{W}$. We make the following remarks: 
1. The solution obtained from the additive decomposition (2.1)-(2.2) above is exact, that is, the solution to $(2.5)-(2.6)$ and $(2.7)-(2.8)$ is the solution to the original fine-scale problem (1.17)-(1.18). This requires, however, the direct sum decomposition (2.3)-(2.4). In practice, the subscale spaces $\tilde{V}$ and $\tilde{W}$ are very difficult-if not impossible-to characterize.

2. The subgrid-scale problem (2.7)-(2.8) is an infinite dimensional, global problem. Therefore, in the form presented above, the complexity of the problem is the same as the original one.

3. The formulation is residual-based in the sense that if the coarse solution is the exact solution, the subscales vanish identically.

4. For the formulation to be advantageous from a computational viewpoint, we need a judicious choice of the coarse and subgrid approximation spaces, as well as a good localization assumption that will decouple the global subgrid problem into a set of local problems. These two key issues are addressed in the remainder of this chapter and in Chapter 3.

\subsection{Choice of finite element spaces}

Although this is by no means necessary, we assume for simplicity that the partitions $\mathcal{T}_{h}$ (fine grid) and $\mathcal{T}_{H}$ (coarse grid) are nested, conforming, and consist of rectangular elements. In other words, the coarse grid $\mathcal{T}_{H}$ results from a Cartesian upgridding of the fine grid $\mathcal{T}_{h}$. The target fine-scale approximation spaces are the lowest-order Raviart-Thomas space for the velocity, $V_{h}=R T_{0}\left(\mathcal{T}_{h}\right)$, and the space of piecewise constants for the pressure, $W_{h}=P_{0}\left(\mathcal{T}_{h}\right)$. We restrict our attention to coarse-scale velocity spaces that are compatible with a piecewise constant approximation of the pressure, $W_{H}=P_{0}\left(\mathcal{T}_{H}\right)$. The two obvious choices are $V_{H}=R T_{0}\left(\mathcal{T}_{H}\right)$ and the BrezziDouglas-Marini space of order $1, V_{H}=B D M_{1}\left(\mathcal{T}_{H}\right)$ [13]. Both spaces satisfy the property $\operatorname{div} V_{H}=W_{H}$, and the inf-sup compatibility condition. In contrast with the 
choice made in [4], here we use the low-order $R T_{0}\left(\mathcal{T}_{H}\right)$ space. Therefore, we define:

$$
\begin{aligned}
V_{H} & =\left\{\boldsymbol{v}_{H}: \boldsymbol{v}_{H}=\sum_{a=1}^{M_{H}} N_{a}^{H} V_{a}, \quad V_{b}=0 \forall \Gamma_{b} \in \Gamma_{u}\right\} \\
W_{H} & =\left\{w_{H}: w_{H}=\sum_{i=1}^{N_{H}} \chi_{i}^{H} W_{i}\right\}
\end{aligned}
$$

where $N_{a}^{H}$ is the $R T_{0}$ basis function associated with the coarse-element face $\Gamma_{a}, V_{a}$ is the integrated coarse-scale flux through $\Gamma_{a}, \chi_{i}^{H}$ is the characteristic function for coarse element $E_{i}$, and $W_{i}$ is the average pressure on coarse element $E_{i}$.

To mimic the fine-scale solution, the subgrid velocities are restricted to belong to the lowest-order Raviart-Thomas space on the fine grid within each coarse element. Let $E_{i, h}=\mathcal{T}_{h}\left(E_{i}\right)$ denote the fine grid defined over coarse element $E_{i}$. The subgridscale velocity field will be defined on each element satisfying the condition:

$$
\tilde{V}_{E_{i}} \subset R T_{0}\left(E_{i, h}\right)
$$

The elements of $\tilde{V}_{E_{i}}$ can be extended to all of $\Omega$ by zero, in order to define the subgrid velocity space as the direct sum of the subgrid spaces over coarse elements:

$$
\tilde{V}=\bigoplus_{i=1}^{N_{H}} \tilde{V}_{E_{i}}
$$

Clearly, in order to have a continuous fine-scale velocity field, the subgrid velocity spaces must satisfy compatibility conditions on the skeleton of the coarse grid. This issue will be discussed in detail in the next subsection.

For consistency, the subgrid pressure space is restricted to belong to the space of discontinuous constant functions on each coarse element:

$$
\tilde{W}_{E_{i}} \subset P_{0}\left(E_{i, h}\right)
$$

An additional condition will be imposed to guarantee uniqueness of the solution. The elements of these spaces are extended by zero functions to the entire domain $\Omega$, and 
the subgrid pressure space is then defined as:

$$
\tilde{W}=\bigoplus_{i=1}^{N_{H}} \tilde{W}_{E_{i}} .
$$

\subsection{Localization of the subgrid problem}

The essential requirement in the construction of our multiscale method is that the approximation be locally conservative at both scales, that is, it must satisfy the discrete version of the mass conservation statement on each element of the coarse and fine grids. This requirement leads to the condition:

$$
\left(w_{H}, \nabla \cdot \boldsymbol{u}_{H}\right)_{E_{i}}=\left(w_{H}, f\right)_{E_{i}} \quad \forall E_{i} \in \mathcal{T}_{H},
$$

or, equivalently, $\nabla \cdot \boldsymbol{u}_{H}=\Pi_{H} f$-the projection of the source term onto the space $W_{H}$ of piecewise constants on the coarse grid. Substituting (2.15) in the coarse-scale equation (2.6) leads to

$$
\left(w_{H}, \nabla \cdot \tilde{\boldsymbol{u}}\right)_{E_{i}}=0 \quad \forall E_{i} \in \mathcal{T}_{H} .
$$

Since $w_{H}$ is constant on each element, we can use the divergence theorem to translate Equation (2.16) into the following compatibility condition on the subgrid velocities:

$$
\int_{\partial E_{i}} \tilde{\boldsymbol{u}} \cdot \boldsymbol{n} \mathrm{d} \Gamma=0 \quad \forall E_{i} \in \mathcal{T}_{H}
$$

Equation (2.17) is the essential condition that guarantees mass conservation at both scales, and allows localizing the subgrid-scale problem. Of course, this condition can be immediately satisfied if one imposes $\tilde{\boldsymbol{u}} \cdot \boldsymbol{n}=0$ on $\partial E_{i}$ pointwise, as was done in [4] and [5], to define the subgrid velocity space on each element as follows:

$$
\tilde{V}_{E_{i}, 0}=\left\{\tilde{\boldsymbol{v}}: \tilde{\boldsymbol{v}} \in R T_{0}\left(E_{i, h}\right), \tilde{\boldsymbol{v}} \cdot \boldsymbol{n}=0 \text { on } \partial E_{i}\right\}
$$


However, this pointwise condition does not account for subgrid velocity variability across element interfaces. The important observation [26] is that such localization assumption is too stringent and, in fact, unnecessary: all that is required is that the subgrid velocities satisfy the weaker compatibility condition (2.17).

As a result, we approximate the global subgrid problem (2.7)-(2.8) as a set of Neumann problems on individual coarse elements. We make the following observations:

1. Since we solve a Neumann problem on each coarse element $E_{i}$, the subgrid velocity test function $\tilde{\boldsymbol{v}}$ must satisfy $\tilde{\boldsymbol{v}} \cdot \boldsymbol{n}=0$ on $\partial E_{i}$, which leads to the following orthogonality condition:

$$
\left(\nabla \cdot \tilde{\boldsymbol{v}}, p_{H}\right)_{E_{i}}=0
$$

2. To guarantee uniqueness of the local subgrid problems, we define the space of subgrid pressure $\tilde{W}$ as the orthogonal complement of $W_{H}$ in $W_{h}$ :

$$
\tilde{W}=W_{H}^{\perp}=\left\{\tilde{w} \in W_{h}:\left(\tilde{w}, w_{H}\right)=0 \forall w_{H} \in W_{H}\right\} .
$$

Since $W_{H}=\operatorname{div} V_{H}$, we have the additional orthogonality relation:

$$
\left(\nabla \cdot \boldsymbol{v}_{H}, \tilde{p}\right)_{E_{i}}=0 \quad \text { or, equivalently } \int_{E_{i}} \tilde{p} \mathrm{~d} \Omega=0
$$

In the light of the observations above, we define the following functional spaces on the fine grid $E_{i, h}$ of each coarse element:

$$
\begin{aligned}
& \tilde{V}_{E_{i}, \tilde{u}}:=\left\{\tilde{\boldsymbol{v}} \in R T_{0}\left(E_{i, h}\right), \tilde{\boldsymbol{v}} \cdot \boldsymbol{n}=\tilde{u} \text { on } \partial E_{i} \text { with } \int_{\partial E_{i}} \tilde{u} \mathrm{~d} \Gamma=0\right\}, \\
& \tilde{W}_{E_{i}}:=\left\{\tilde{w} \in P_{0}\left(E_{i, h}\right), \int_{E_{i}} \tilde{w} \mathrm{~d} \Omega=0\right\} .
\end{aligned}
$$

The global subgrid spaces are defined through an extension to $\Omega$ by zero functions and a direct sum like in Equations (2.12) and (2.14). 


\subsection{Boundary conditions for the local subgrid prob- lems}

Using the compatibility condition (2.17) and the orthogonality conditions (2.19)(2.21), the subgrid problem reads: For each coarse element $E_{i}=1, \ldots, N_{H}$, find $(\tilde{\boldsymbol{u}}, \tilde{p}) \in \tilde{V}_{E_{i}, \tilde{u}} \times \tilde{W}_{E_{i}}$ such that

$$
\begin{aligned}
\left(\tilde{\boldsymbol{v}}, \mathbf{k}^{-1} \tilde{\boldsymbol{u}}\right)_{E_{i}}- & (\nabla \cdot \tilde{\boldsymbol{v}}, \tilde{p})_{E_{i}}=-\left(\tilde{\boldsymbol{v}}, \mathbf{k}^{-1} \boldsymbol{u}_{H}\right)_{E_{i}} \\
(\tilde{w}, \nabla \cdot \tilde{\boldsymbol{u}})_{E_{i}} & =\left(\tilde{w}, f-\nabla \cdot \boldsymbol{u}_{H}\right)_{E_{i}}
\end{aligned}
$$

for all $\tilde{\boldsymbol{v}} \in \tilde{V}_{E_{i}, 0}$ and $\tilde{w} \in \tilde{W}_{E_{i}}$. Given $\boldsymbol{u}_{H}$ and the local boundary conditions $\tilde{u}_{E_{i}}$ (to be discussed next), the problem above has a unique solution. The global subgrid scale solution $(\tilde{\boldsymbol{u}}, \tilde{p})$ is then obtained by patching together the solutions on each coarse element.

In the remaining of this section, we shall restrict our attention to the case when the source function does not display a multiscale character. Mathematically, this means that the source function is equal to its projection on the space of coarse-scale pressures:

$$
\left(w_{H}, f\right)=\left(w_{H}, 1\right) f \quad \text { for all } w_{H} \in W_{H}
$$

or, equivalently,

$$
f=\Pi_{H} f=f_{H}=\nabla \cdot u_{H}
$$

Under these conditions, the right-hand side of Equation (2.25) is equal to zero. Clearly, the case in which equation (2.27) is not satisfied is important in the presence of wells, and it is discussed at length in Chapter 3.

\subsubsection{No subgrid communication}

Consider first the choice of zero subgrid flux across coarse elements, $\tilde{u}=0$ on $\partial E$ for all $E \in \mathcal{T}_{H}[4,5]$. Recalling Equation (2.9), the subgrid solution inside element $E$ 
takes the form:

$$
\begin{aligned}
& \tilde{\boldsymbol{u}}=\sum_{a} \tilde{\boldsymbol{N}}_{a}^{0} U_{a}, \\
& \tilde{p}=\sum_{a} \tilde{\phi}_{a}^{0} U_{a} .
\end{aligned}
$$

where $\left(\tilde{N}_{a}^{0}, \tilde{\phi}_{a}^{0}\right) \in \tilde{V}_{E, 0} \times \tilde{W}_{E}$ is the solution to:

$$
\begin{gathered}
\left(\tilde{\boldsymbol{v}}, \mathbf{k}^{-1} \tilde{\boldsymbol{N}}_{a}^{0}\right)_{E}-\left(\nabla \cdot \tilde{\boldsymbol{v}}, \tilde{\phi}_{a}^{0}\right)_{E}=-\left(\tilde{\boldsymbol{v}}, \mathbf{k}^{-1} \boldsymbol{N}_{a}^{H}\right)_{E} \quad \forall \tilde{\boldsymbol{v}} \in \tilde{V}_{E, 0} \\
\left(\tilde{w}, \nabla \cdot \tilde{\boldsymbol{N}}_{a}^{0}\right)_{E}=0 \quad \forall \tilde{w} \in \tilde{W}_{E}
\end{gathered}
$$

This solution is referred to as a subgrid numerical Green function [4]. Equations (2.28)(2.29) show that the subgrid solution scales linearly with the coarse-scale fluxes $U_{a}$.

\subsubsection{Proposed subgrid communication}

If subgrid communication between coarse elements is disallowed, the subgrid velocity is restricted to recirculation functions within each coarse element. This localization assumption clearly limits the ability of the method to capture thin but long-range permeability structures that span more than one coarse element. In general, if $\tilde{u} \neq$ 0 on $\partial E$, the subgrid solution takes the form:

$$
\begin{aligned}
& \tilde{\boldsymbol{u}}=\tilde{\boldsymbol{u}}_{\text {skel }}+\sum_{a} \tilde{\boldsymbol{N}}_{a}^{0} U_{a} \\
& \tilde{p}=\tilde{p}_{\text {skel }}+\sum_{a} \tilde{\phi}_{a}^{0} U_{a}
\end{aligned}
$$

where $\left(\tilde{\boldsymbol{u}}_{\text {skel }}, \tilde{p}_{\text {skel }}\right)$ reflects the influence of the local subgrid boundary conditions. We design these to satisfy the following conditions:

1. Lead to a flux-continuous (conforming) velocity field.

2. Result in a discretization that is locally mass-conservative at both scales.

3. Reflect the fine-scale heterogeneity. 
4. Be computable without knowledge of the global velocity field.

Condition (1) will be satisfied if the subgrid flux is uniquely defined on the skeleton $\mathcal{S}_{H}$ of the coarse grid $\mathcal{T}_{H}$. An easy way to ensure condition (2) is to impose that the subscale flux satisfy:

$$
\int_{\Gamma_{a}} \tilde{u} \mathrm{~d} \Gamma=0 \quad \forall \Gamma_{a} \in \mathcal{S}_{H}
$$

Condition (3) requires that the subscale velocity be solution to local problems using the fine-scale permeability $\mathbf{k}$. In an attempt to satisfy condition (4), we let the subgrid flux on each coarse edge scale with the coarse flux through that edge. Equations $(2.32)-(2.33)$ are then re-written as:

$$
\begin{aligned}
& \tilde{\boldsymbol{u}}=\sum_{a}\left(\tilde{\boldsymbol{N}}_{a}^{\mathrm{skel}}+\tilde{\boldsymbol{N}}_{a}^{0}\right) U_{a}=\sum_{a} \tilde{\boldsymbol{N}}_{a} U_{a}, \\
& \tilde{p}=\sum_{a}\left(\tilde{\phi}_{a}^{\text {skel }}+\tilde{\phi}_{a}^{0}\right) U_{a}=\sum_{a} \tilde{\phi}_{a} U_{a} .
\end{aligned}
$$

Therefore, we can define the multiscale velocity as the sum of the coarse-scale and subgrid-scale components:

$$
\boldsymbol{u}_{H, h}=\boldsymbol{u}_{H}+\tilde{\boldsymbol{u}}=\sum_{a}\left(\boldsymbol{N}_{a}^{H}+\tilde{\boldsymbol{N}}_{a}\right) U_{a}=\sum_{a} \boldsymbol{N}_{a}^{H, h} U_{a}
$$

where $N_{a}^{H, h}$ is the multiscale velocity basis function associated with interface $\Gamma_{a}$. Different definitions of the multiscale basis functions exist [16, 1, 2], and in this work we have adopted the recent one proposed by [28], where the multiscale basis function is the solution to a flow problem restricted to a pair of adjacent coarse elements with source terms specified in such a way that the flow through the interface is identically one (see Figure 2-1). More precisely, the multiscale basis functions $\left(N_{a}^{H, h}, \phi_{a}^{H, h}\right)$ for interface $\Gamma_{a}$ (common to coarse elements $E_{i}$ and $E_{j}$ ) are the solution to the following 
local problem:

$$
\begin{aligned}
\mathbf{k}^{-1} \boldsymbol{N}_{a}^{H, h}+\nabla \phi_{a}^{H, h} & =0 \quad \text { in } E_{i} \cup E_{j}, \\
\nabla \cdot \boldsymbol{N}_{a}^{H, h} & = \begin{cases}w(x) / \int_{E_{i}} w(x) \mathrm{d} \Omega & \text { if } x \in E_{i}, \\
-w(x) / \int_{E_{j}} w(x) \mathrm{d} \Omega & \text { if } x \in E_{j},\end{cases} \\
\boldsymbol{N}_{a}^{H, h} \cdot \boldsymbol{n} & =0 \quad \text { on } \partial\left(E_{i} \cup E_{j}\right) .
\end{aligned}
$$

In [28], it is suggested that the source function $w(x)$ be scaled with the trace of the permeability tensor, $\operatorname{trace} \mathbf{k}(x)$. To ensure that the method is mass conservative at the coarse and subscale level, we impose the following scaling for $w(x)$ on each element:

$$
w(x)= \begin{cases}\operatorname{trace} \mathbf{k}(x) & \text { if } \Pi_{H} f=0 \\ 1 & \text { if } \Pi_{H} f \neq 0\end{cases}
$$

It is important to observe that the coarse-scale $R T_{0}$ basis functions are in fact solutions to $(2.38)-(2.41)$ with $\mathbf{k}(x)=$ constant and $w(x)=1$.

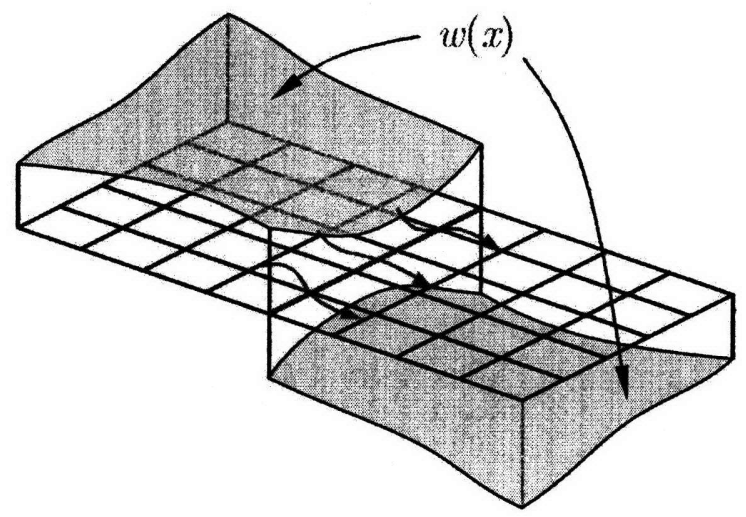

Figure 2-1: Diagram illustrating the local flow problem defining the multiscale basis functions.

Remark. The local problem (2.38)-(2.40) can be solved using a low-order $\left(R T_{0}\right)$ mixed finite element method, or a finite volume method. Clearly, for constant $\mathbf{k}$, the multiscale basis function reduces to the common $R T_{0}$ basis function, and the subgrid 
basis function is identically equal to zero. In the presence of subgrid heterogeneity, the subgrid basis function will capture not only flow redistribution within the coarse element, but also preferential flow across the interface.

Proposition. If we define the subscale basis functions on each element $E_{i}$ as:

$$
\begin{aligned}
& \tilde{N}_{a}=N_{a}^{H, h}-N_{a}^{H}, \\
& \tilde{\phi}_{a}^{E_{i}}=\phi_{a}^{H, h}-\frac{1}{\left|E_{i}\right|} \int_{E_{i}} \phi_{a}^{H, h} \mathrm{~d} \Omega,
\end{aligned}
$$

then

$$
\begin{gathered}
\tilde{\boldsymbol{u}}=\sum_{a} \tilde{\boldsymbol{N}}_{a} U_{a}, \\
\tilde{p}=\sum_{a} \tilde{\phi}_{a}^{E_{i}} U_{a},
\end{gathered}
$$

is a solution to the subgrid problem (2.24)-(2.25).

Proof. We first show that the solution (2.44)-(2.45) satisfies the mass balance equation (2.25). Recall that we consider the case with a source function $f=\Pi_{H} f$ only. The case $f \notin W_{H}$ will be treated in Section 3. Under this assumption, Equation (2.25) reduces to

$$
(\tilde{w}, \nabla \cdot \tilde{\boldsymbol{u}})_{E}=0 \quad \forall \tilde{w} \in \tilde{W}_{E}
$$

or, equivalently,

$$
\sum_{a} U_{a}\left(\tilde{w}, \nabla \cdot \tilde{N}_{a}\right)_{E}=0 \quad \forall \tilde{w} \in \tilde{W}_{E}
$$

We must consider two cases:

1. Zero source on element $E, \Pi_{H} f=0$. In this case, mass conservation on the coarse scale implies

$$
\nabla \cdot \boldsymbol{u}_{H}=0 \text { on } E \Rightarrow \int_{\partial E} \boldsymbol{u}_{H} \cdot \boldsymbol{n} \mathrm{d} \Gamma=0 \Rightarrow \sum_{a} U_{a}=0
$$

Under this condition, Equation (2.47) is satisfied as long as $\nabla \cdot \tilde{\boldsymbol{N}}_{a}$ is a function 
of position, but independent of the face $a$. This is indeed satisfied by our definition of the multiscale basis functions with $w(x)$ given by Equation (2.41).

2. Nonzero source on element $E, \Pi_{H} f \neq 0$. In this case, Equation (2.48) does not hold, and Equation (2.47) implies

$$
\left(\tilde{w}, \nabla \cdot \tilde{N}_{a}\right)_{E}=0 \quad \forall \tilde{w} \in \tilde{W}_{E}
$$

for all $\Gamma_{a} \in \partial E$ individually. This condition, together with Equation 2.34, implies that $\nabla \cdot \tilde{N}_{a} \equiv 0$ on $E$. Once again, this is satisfied by our definition of the source function in Equation (2.41).

We now need to show that $(\tilde{\boldsymbol{u}}, \tilde{p})$ defined in Equations (2.44)-(2.45) satisfy the Darcy equation (2.24). First we note that, by virtue of (2.42)-(2.43),

$$
\int_{\Gamma_{a}} \tilde{\boldsymbol{N}}_{a} \cdot \boldsymbol{n} \mathrm{d} \Gamma=0, \quad \int_{E} \tilde{\phi}_{a}^{E} \mathrm{~d} \Omega=0
$$

Therefore, $(\tilde{\boldsymbol{u}}, \tilde{p}) \in \tilde{V}_{E, \tilde{u}} \times \tilde{W}_{E}$ independently of the coarse-scale fluxes $U_{a}$. Using integration by parts, we write Equation (2.24) in strong form:

$$
\mathbf{k}^{-1} \tilde{\boldsymbol{u}}+\nabla \tilde{p}=-\mathbf{k}^{-1} \boldsymbol{u}_{H} \text { in } E
$$

or, equivalently:

$$
\sum_{a} U_{a}\left(\mathbf{k}^{-1} \boldsymbol{N}_{a}^{H, h}+\nabla \phi_{a}^{H, h}\right)=0 \text { in } E .
$$

Indeed, the term in parenthesis is identically equal to zero by Equation (2.38). This completes the proof.

Remark. One of the main features of the proposed method is the relaxed localization assumption with respect to the numerical subgrid upscaling method [4]. We do not provide direct numerical comparisons between the two methods, for two reasons. First, results in [4] use a higher-order velocity space $\left(B D M_{1}\right)$ on the coarse scale, while we use the lowest-order space $\left(R T_{0}\right)$-therefore, a fair direct comparison is difficult. 
We point out, however, that the choice of $R T_{0}$ in the numerical subgrid upscaling method would imply that the subscales are identically equal to zero-clearly a limitation. Second, in the paper we show that, in the absence of multiscale source terms, our method is equivalent to a multiscale mixed finite element method [2]. A thorough comparison of different multiscale techniques (including the numerical subgrid upscaling method) is provided by [28].

A slightly modified problem needs to be solved for the multiscale basis function at a face $\Gamma_{a}$ on the boundary of the domain. If $\Gamma_{a} \subset \Gamma_{p}$, we solve the same local problem (2.38)-(2.40), with an imaginary coarse element whose permeability is a reflection (with respect to $\Gamma_{a}$ ) of the permeability of the element inside the domain. Although our description in this paper is restricted to the case of zero prescribed flux, $\vec{u}=0$, our implementation certainly allows for $\bar{u} \neq 0$. In that case, if $\Gamma_{a} \subset \Gamma_{u}$, we solve the following local problem:

$$
\begin{aligned}
\mathbf{k}^{-1} \boldsymbol{N}_{a}^{H, h}+\nabla \phi_{a}^{H, h} & =0 \quad \text { in } E_{i}, \\
\nabla \cdot \boldsymbol{N}_{a}^{H, h} & =-w(x) / \int_{E_{i}} w(x) \mathrm{d} \Omega, \\
\boldsymbol{N}_{a}^{H, h} \cdot \boldsymbol{n} & = \begin{cases}0 & \text { on } \partial E_{i} \backslash \Gamma_{a}, \\
\bar{u} / \int_{\Gamma_{a}} \bar{u} \mathrm{~d} \Gamma & \text { on } \Gamma_{a},\end{cases}
\end{aligned}
$$

We note that the multiscale basis function may differ from the $R T_{0}$ coarse basis function due to subgrid variability of either the permeability $\mathbf{k}$ or the prescribed flux $\bar{u}$. Our definition ensures that the fine-scale boundary fluxes are honored exactly. 


\subsection{The coarse-scale problem revisited}

Making use of the compatibility condition (2.16) and the orthogonality relation (2.21), the coarse-scale problem (2.5)-(2.6) reads: Find $\left(\boldsymbol{u}_{H}, p_{H}\right) \in V_{H} \times W_{H}$ such that

$$
\begin{aligned}
\left(\boldsymbol{v}_{H}, \mathbf{k}^{-1} \boldsymbol{u}_{H}\right)+\left(\boldsymbol{v}_{H}, \mathbf{k}^{-1} \tilde{\boldsymbol{u}}\right)-\left(\nabla \cdot \boldsymbol{v}_{H}, p_{H}\right) & =-\left\langle\boldsymbol{v}_{H} \cdot \boldsymbol{n}, \bar{p}\right\rangle_{\Gamma_{p}}, \\
\left(w_{H}, \nabla \cdot \boldsymbol{u}_{H}\right) & =\left(w_{H}, f\right),
\end{aligned}
$$

for all $\boldsymbol{v}_{H} \in V_{H}$ and $w_{H} \in W_{H}$. It is interesting to note that the the subgrid contribution to the coarse-scale problem, albeit essential, is confined to the second term of Equation (2.56).

This form of the problem is nonsymmetric. In practice, it is convenient to express it as an equivalent, symmetric problem. For this purpose, we define the multiscale velocity and pressure space functions:

$$
\begin{gathered}
V_{H, h}=\left\{\boldsymbol{v}_{H, h}: \boldsymbol{v}_{H, h}=\sum_{a=1}^{M_{H}} \boldsymbol{N}_{a}^{H, h} V_{a}, \quad V_{b}=0 \forall \Gamma_{b} \in \Gamma_{u}\right\}, \\
W_{H, h}=W_{H} \oplus \tilde{W}=\left\{w_{H, h}: w_{H, h}=\sum_{i=1}^{N_{H}} \chi_{i}^{H}\left(W_{i}+\sum_{a} \tilde{\phi}_{a}^{E_{i}} V_{a}\right)\right\},
\end{gathered}
$$

Proposition. The coarse scale problem (2.56)-(2.57) can be written in the following equivalent, symmetric form: Find $\left(\boldsymbol{u}_{H, h}, p_{H, h}\right) \in V_{H, h} \times W_{H, h}$ such that

$$
\begin{aligned}
\left(\boldsymbol{v}_{H, h}, \mathbf{k}^{-1} \boldsymbol{u}_{H, h}\right)-\left(\nabla \cdot \boldsymbol{v}_{H, h}, p_{H, h}\right) & =-\left\langle\boldsymbol{v}_{H, h} \cdot \boldsymbol{n}, \bar{p}\right\rangle_{\Gamma_{p}} \\
\left(w_{H, h}, \nabla \cdot \boldsymbol{u}_{H, h}\right) & =\left(w_{H, h}, f\right)
\end{aligned}
$$

for all $\boldsymbol{v}_{H, h} \in V_{H, h}$ and $w_{H, h} \in W_{H, h}$.

Proof. We first show that Equations (2.56) and (2.60) are equivalent. The first term in $(2.60)$ can be written as:

$$
\left(\boldsymbol{v}_{H, h}, \mathbf{k}^{-1} \boldsymbol{u}_{H, h}\right)=\left(\boldsymbol{v}_{H}, \mathbf{k}^{-1} \boldsymbol{u}_{H, h}\right)+\left(\tilde{\boldsymbol{v}}, \mathbf{k}^{-1} \boldsymbol{u}_{H, h}\right)
$$


Exploiting the orthogonality properties $\operatorname{div} V_{H} \perp \tilde{W}$ and $\operatorname{div} \tilde{V} \perp W_{H}$, and integration by parts, the second term reads:

$$
-\left(\nabla \cdot \boldsymbol{v}_{H, h}, p_{H, h}\right)=-\left(\nabla \cdot \boldsymbol{v}_{H}, p_{H}\right)+(\tilde{\boldsymbol{v}}, \nabla \tilde{p})-\langle\tilde{\boldsymbol{v}} \cdot \boldsymbol{n}, \bar{p}\rangle_{\Gamma_{p}} .
$$

Inserting the two equations above in Equation (2.60), we obtain:

$$
\left(\boldsymbol{v}_{H}, \mathbf{k}^{-1} \boldsymbol{u}_{H, h}\right)-\left(\nabla \cdot \boldsymbol{v}_{H}, p_{H}\right)+\left(\tilde{\boldsymbol{v}}, \mathbf{k}^{-1} \boldsymbol{u}_{H, h}+\nabla \tilde{p}\right)=-\left\langle\boldsymbol{v}_{H} \cdot \boldsymbol{n}, \bar{p}\right\rangle_{\Gamma_{p}}
$$

The third term in the equation above vanishes due to our definition of the subgrid problem, so we have arrived at Equation (2.56).

We now show the equivalence of Equations (2.57) and (2.61). Exploiting the orthogonality properties once again, and recalling that in this section we are considering the case $f=\Pi_{H} f$ only, Equation (2.61) takes the form:

$$
\left(w_{H}, \nabla \cdot \boldsymbol{u}_{H}\right)+(\tilde{w}, \nabla \cdot \tilde{\boldsymbol{u}})=\left(w_{H}, f\right)
$$

The second term vanishes due to the imposed mass conservation at the fine scale (see Equation (2.46) and the subsequent discussion). This completes the proof.

\subsection{A multiscale method with a coarse pressure approximation}

The proposition above shows that, for the case in which the source term does not display fine-scale variability, our variational multiscale mixed finite element (VMSMFE) method is equivalent to a multiscale mixed finite element method. This observation was made in [6] for the case when subgrid communication was disallowed (they also treated a method with oversampling that leads to a nonconforming fine-scale velocity field). Numerical results from the solution of Equations (2.56)-(2.57) (or their symmetric equivalent (2.60)-(2.61)) were given in [26]. Further experimentation has shown that improved results are obtained when the pressure space is restricted to 
belong to the space of piecewise constant functions on the coarse grid.

The problem to be solved is essentially identical to (2.60)-(2.61), except that the solution space for the pressure is $W_{H}$ instead of $W_{H, h}$ : Find $\left(\boldsymbol{u}_{H, h}, p_{H}\right) \in V_{H, h} \times W_{H}$ such that

$$
\begin{aligned}
\left(\boldsymbol{v}_{H, h}, \mathbf{k}^{-1} \boldsymbol{u}_{H, h}\right)-\left(\nabla \cdot \boldsymbol{v}_{H, h}, p_{H}\right) & =-\left\langle\boldsymbol{v}_{H, h} \cdot \boldsymbol{n}, \bar{p}\right\rangle_{\Gamma_{p}} \\
\left(w_{H}, \nabla \cdot \boldsymbol{u}_{H, h}\right) & =\left(w_{H}, f\right)
\end{aligned}
$$

for all $\boldsymbol{v}_{H, h} \in V_{H, h}$ and $w_{H} \in W_{H}$.

In the absence of a source term with multiscale character, this method is precisely the multiscale mixed finite element method proposed in [28]. Our method differs in its derivation (the variational multiscale framework rather than the multiscale finite element method) and, more importantly, in the treatment of fine-scale sources. The improved behavior is not restricted to the pressure solution, but also the velocity field, since both fields are coupled. In any case, the fine-scale pressure can still be reconstructed according to the multiscale decomposition (2.2) with $\tilde{p}$ given by Equation (2.45) once the solution has been computed. This step does not carry any additional computational cost, as the subgrid-scale pressure basis functions are obtained together with the velocity basis functions.

\subsubsection{Implementation}

The implementation of the VMSMFE method is relatively straightforward. It consists of the following steps:

1. Precompute the multiscale basis functions $\left(\boldsymbol{N}_{a}^{H, h}, \phi_{a}^{H, h}\right)$ for each coarse interface $\Gamma_{a}$.

2. Build the system of equations corresponding to the coarse-scale problem (2.66)(2.67):

$$
\left[\begin{array}{cc}
\mathbf{A} & -\mathbf{B}^{t} \\
\mathbf{B} & \mathbf{0}
\end{array}\right]\left[\begin{array}{l}
\mathbf{U} \\
\mathbf{P}
\end{array}\right]=\left[\begin{array}{l}
\mathbf{g} \\
\mathbf{f}
\end{array}\right],
$$


to be solved for the coarse-scale interface fluxes $\mathbf{U}=\left\{U_{a}\right\}$ and cell-center pressures $\mathbf{P}=\left\{P_{i}\right\}$. The system incorporates the subgrid-scale contributions in matrix A, obtained by assembly of the coarse-element contributions:

$$
A_{a b}^{E}=\int_{E} \boldsymbol{N}_{a}^{H, h} \mathbf{k}^{-1} \boldsymbol{N}_{b}^{H, h} \mathrm{~d} \Omega .
$$

Since both the permeability tensor and the basis functions display a multiscale character, the integrals must be evaluated on the underlying fine grid. The matrix is symmetric, since it corresponds to the symmetric form of the problem.

3. Reconstruct the fine-scale velocity and pressure fields resorting to the additive decomposition (2.1)-(2.2). The subgrid part is obtained by linear combination of the (known) coarse-scale fluxes and (precomputed) subgrid-scale basis functions. 


\section{Chapter 3}

\section{Multiscale source terms}

In this section, we shall examine the case when the source/sink term presents a multiscale character. This is a scenario of paramount importance because, in practice, flow is driven by injection and production wells. Wells are features that are much smaller than the resolution that one can afford in field-scale simulations and should, therefore, be understood as concentrated (point or line) sources. A well model relates the flow rate into or out of the well with the difference between the wellbore pressure and the average pressure of the well gridblock. An analytical representation of such relationship is possible in simplified cases-in particular, when the well block is assumed to be homogeneous $[31,8]$. In a multiscale method that attempts to capture subgrid variability, two things are needed:

1. A computational strategy to handle concentrated sources with variability at the scale of the fine grid.

2. A well model that relates flow rate with the pressure difference between the wellbore and the fine well block.

In this paper, we concentrate exclusively on the first issue, and assume that the source term is a function that displays variability at the scale of the fine grid. In particular, we shall assume that the source term $f$ belongs to the space of piecewise constant functions on the fine grid:

$$
f=\Pi_{h} f \in W_{h} .
$$


To fully account for the presence of wells, standard well models can then be used at the fine scale.

Since this is a case of practical interest, we shall understand that $f$ consists of a number of concentrated sources: ${ }^{1}$

$$
f(x)=\sum_{j=1}^{N_{w}} f_{j}(x) .
$$

Each $f_{j}$ is a concentrated source/sink that is constant on a fine scale gridblock $e_{j}$, inside a coarse block $E_{j}$. Of course, several wells may exist within the same coarse block. In Section 2 we presented a variational multiscale method for the solution of the problem without fine wells, that is, when the sources where assumed to be spread over coarse elements, $f=\Pi_{H} f=0$. In this section, we extend the formulation to consider subgrid variations of the source term,

$$
\tilde{f}:=f-\Pi_{H} f \neq 0
$$

while preserving mass conservation at both coarse and subgrid scales.

\subsection{Principle}

We start by decomposing the source term into its coarse scale and subscale components:

$$
f=f_{H}+\tilde{f}
$$

where $f_{H} \in W_{H}$ and $\tilde{f} \in \tilde{W}$. The subscale component is simply the deviatoric (zeromean) part of $f$ on each coarse element. It is then natural to decompose the full

\footnotetext{
${ }^{1}$ In what follows, we shall abuse language and refer to 'wells' when, strictly speaking, we mean 'concentrated or point sources'.
} 
multiscale solution as follows:

$$
\begin{aligned}
& \boldsymbol{u}_{H, h}^{\text {well }}=\boldsymbol{u}_{H, h}+\boldsymbol{u}^{\text {well }} \\
& p_{H, h}^{\text {well }}=p_{H, h}+p^{\text {well }}
\end{aligned}
$$

where $\left(\boldsymbol{u}_{H, h}, p_{H, h}\right)$ is the solution to a problem with coarse source terms $f_{H}$, and $\left(\boldsymbol{u}^{\text {well }}, p^{\text {well }}\right)$ is the solution to a problem with deviatoric fine-scale sources $\tilde{f}$. Special care must be taken of the prescribed-pressure boundary conditions. As we will see, the well problem is defined as a Neumann problem with deviatoric sources on each element, and zero-flux boundary conditions. This results in certain pressures at the boundary, $\bar{p}^{\text {well }}$. The multiscale solution must then be seen as the solution to the original problem, but with coarse sources and with pressure boundary conditions replaced by $\bar{p}-\bar{p}^{\text {well }}$.

\subsection{The local well problem}

Recall the decomposition of the source term into individual wells $j=1, \ldots, N_{w}$. We express the well solution as

$$
\begin{aligned}
\boldsymbol{u}^{\text {well }} & =\sum_{j=1}^{N_{w}} Q_{j} \psi_{j}^{\text {well }} \\
p^{\text {well }} & =\sum_{j=1}^{N_{w}} Q_{j} \varphi_{j}^{\text {well }}
\end{aligned}
$$

where $Q_{j}$ is the strength of each well:

$$
Q_{j}=\int_{e_{j}} f(x) \mathrm{d} x=f_{j}\left|e_{j}\right|
$$

and $\psi_{j}^{\text {well }}$ and $\phi_{j}^{\text {well }}$ are the well velocity and pressure basis functions.

In order to maintain the computational complexity of the multiscale method, we assume that the well functions have local support in a subdomain $\Omega_{j}$ that is 
equal to or larger than coarse block $E_{j}$ containing the well, that is, $e_{j} \subset E_{j} \subseteq \Omega_{j}$. More precisely, the well basis functions $\left(\psi_{j}^{\text {well }}, \varphi_{j}^{\text {well }}\right)$ for well $j$ are the solution to the following problem:

$$
\begin{aligned}
& \mathbf{k}^{-1} \boldsymbol{\psi}_{j}^{\text {well }}+\nabla \varphi_{j}^{\text {well }}=0 \quad \text { in } \Omega_{j} \\
& \nabla \cdot \boldsymbol{\psi}_{j}^{\text {well }}= \begin{cases}\frac{1}{\left|e_{j}\right|}-\frac{1}{\left|E_{j}\right|} & \text { if } x \in e_{j} \\
-\frac{1}{\left|E_{j}\right|} & \text { if } x \in E_{j} \backslash e_{j} \\
0 & \text { if } x \in \Omega_{j} \backslash E_{j}\end{cases} \\
& \boldsymbol{\psi}_{j}^{\text {well }} \cdot \boldsymbol{n}=0 \quad \text { on } \partial \Omega_{j} \text {. }
\end{aligned}
$$

From an implementation standpoint, the well region $\Omega_{j}$ is a group of elements surrounding the fine well block $e_{j}$ (see Figure 3-1). The actual size of $\Omega_{j}$ is determined by setting a length scale parameter $L_{w}$. We choose to express this length scale as a fraction $N_{l}$ of the characteristic dimension of the domain, $L_{\Omega}$, such that:

$$
L_{w}=N_{l} L_{\Omega}
$$

Clearly, a larger $L_{w}$ will allow for a more accurate representation of the well effects, at the cost of increasing the computational cost. In the numerical simulations of the next section, we typically choose values between $N_{l}=1 / 16$ and $N_{l}=1 / 4$. Near boundaries, the well region is always restricted to lie inside the domain. 


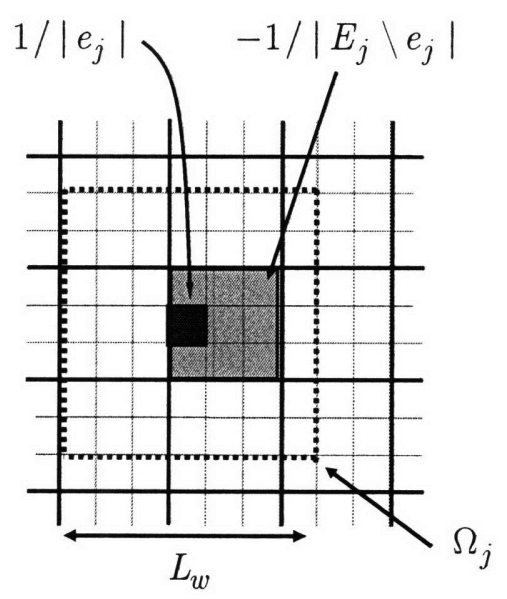

Figure 3-1: Diagram illustrating the local flow problem defining the well basis functions.

Remark. The proposed approach to handle concentrated sources is fundamentally different from the one proposed in [35]. Both approaches superpose two solutions: a well solution, and a background solution. The split in [35] is succinctly expressed in a paragraph from their paper: "... the source term is removed from the coarse cell that contains the well; the well effects, which are captured by the well basis function, are represented on the coarse scale as integral contributions to adjacent coarse cells." In contrast, our background solution contains the entire strength of the source term, albeit averaged (constant over the well gridblock). The well contribution (more generally, the contribution from any multiscale source term) is the solution to a local problem with zero integral divergence-purely a deviatoric (or zero-mean) source term. Our treatment effectively removes the well singularity from the coarsescale problem, is naturally mass conservative, and allows well regions to overlap.

\subsection{The coarse-scale problem revisited}

The solution of the fine-scale well problem as proposed here is independent of the multiscale problem with coarse source terms. Once the well basis functions $\left(\boldsymbol{\psi}_{j}^{\text {well }}, \varphi_{j}^{\text {well }}\right)$ have been computed for all wells and the fine-scale well solution constructed by linear combination through Equations (3.7)-(3.8), the pressure at the boundary $\bar{p}^{\text {well }}$ is 
recorded.

The coarse-scale problem (2.66)-(2.67) must be modified only to replace the source function by its gridblock-averaged counterpart, and to incorporate the (subgrid-scale) influence of wells on boundaries with prescribed pressure.

The coarse-scale problem reads: Find $\left(\boldsymbol{u}_{H, h}, p_{H}\right) \in V_{H, h} \times W_{H}$ such that

$$
\begin{aligned}
\left(\boldsymbol{v}_{H, h}, \mathbf{k}^{-1} \boldsymbol{u}_{H, h}\right)-\left(\nabla \cdot \boldsymbol{v}_{H, h}, p_{H}\right) & =-\left\langle\boldsymbol{v}_{H, h} \cdot \boldsymbol{n}, \bar{p}-\bar{p}^{\text {well }}\right\rangle_{\Gamma_{p}} \\
\left(w_{H}, \nabla \cdot \boldsymbol{u}_{H, h}\right) & =\left(w_{H}, f_{H}\right)
\end{aligned}
$$

for all $\boldsymbol{v}_{H, h} \in V_{H, h}$ and $w_{H} \in W_{H}$. Once the coarse-scale problem has been computed, the full solution is given by (3.5)-(3.6).

This formulation-based on introducing additional basis functions associated with each well-removes the singularities from the multiscale problem due to the presence of wells. Our definition of the (local) well problems as Neumann problems with a deviatoric source in the coarse well block leads to a mass conservative velocity at both scales. An important feature of our formulation is that well regions are allowed to overlap without compromising mass conservation or flux continuity, which gives flexibility with regard to the choice of the well length scale. 


\section{Chapter 4}

\section{Numerical simulations}

In this section we illustrate the performance of the VMSMFE method in several cases of increasing complexity. We restrict our attention to examples in two dimensions with uniform rectangular grids. To assess the accuracy and robustness of the method with respect to heterogeneity, meshes and wells, we compare the solutions obtained using the proposed multiscale method with the reference fine-scale solution obtained using a two-point finite volume method.

We compute a mean pressure error in the following manner:

$$
\varepsilon(p)=\frac{\left\|\mathbf{p}-\mathbf{p}^{\text {ref }}\right\|^{2}}{\left\|\mathbf{p}^{\text {ref }}\right\|^{2}},
$$

where $p$ and $p^{\text {ref }}$ are array vectors that contain the average pressure in each fine element (multiscale solution and reference finite volume solution, respectively), and $\|\cdot\|$ is the usual discrete $l^{2}$ norm. The velocity error is computed as:

$$
\varepsilon(\boldsymbol{u})=\frac{\left\|\mathrm{u}_{x}-\mathbf{u}_{x}^{\text {ref }}\right\|^{2}}{\left\|\mathbf{u}_{x}^{\text {ref }}\right\|^{2}}+\frac{\left\|\mathrm{u}_{y}-\mathbf{u}_{y}^{\text {ref }}\right\|^{2}}{\left\|\mathbf{u}_{y}^{\text {ref }}\right\|^{2}}
$$

where $u_{x}$ and $u_{y}$ are array vectors that contain the average velocities across the fine mesh interfaces in the $x$ - and $y$-directions. 


\subsection{Small channelized system}

The first example illustrates the ability of the method to capture the global and detailed flow pattern in the presence of drastic subgrid heterogeneity. The fine grid is just a $4 \times 4$ grid, with an isotropic permeability field shown in Figure 4-1(a). The white blocks are highly conductive $(k=1)$ and the gray blocks correspond to low permeability $\left(k=10^{-3}\right)$. Flow is left-to-right, with the left boundary set at a pressure $\bar{p}_{\text {left }}=1$ and the right boundary at $\bar{p}_{\text {right }}=0$. The top and bottom boundaries are no-flow boundaries.

The pressure and flux in the $x$-direction from a finite volume solution computed on the fine grid are shown in Figure 4-1. The contours of $x$-flux clearly indicate the preferential flow path along the high-conductivity channel. In Figure 4-2 we show the solution obtained using the VMSMFE method on a coarse grid of $2 \times 2$ elements. The multiscale solution captures the sharp contrast in permeability even though there is no scale separation at all (the high-permeability channel spans the entire domain).

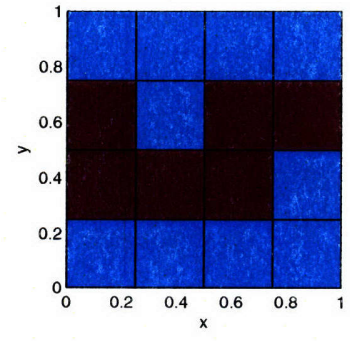

(a) permeability

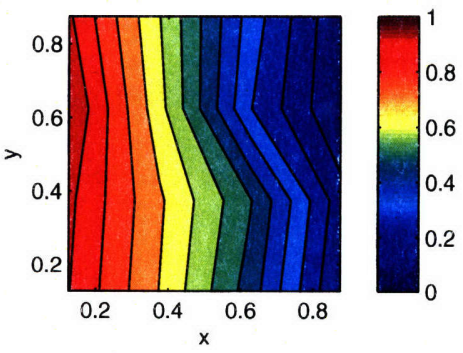

(b) pressure

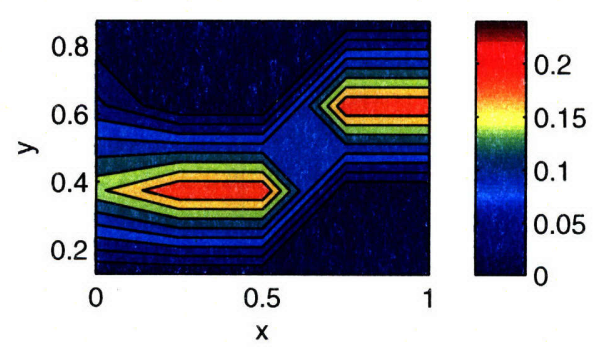

(c) flux in $x$-direction

Figure 4-1: Small channelized system. Fine-scale finite volume solution. 


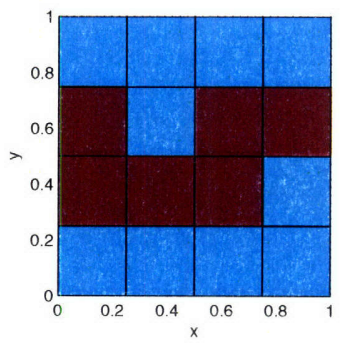

(a) permeability

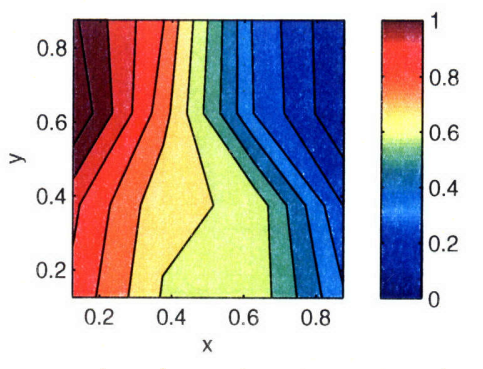

(b) pressure

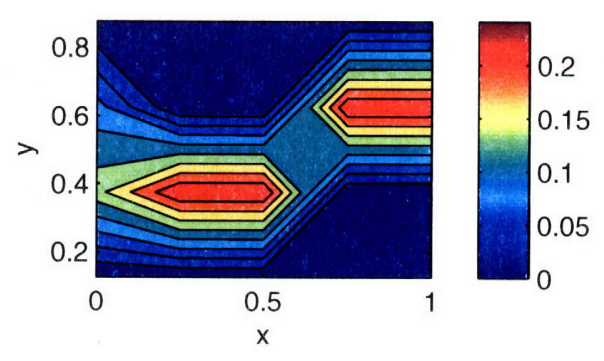

(c) flux in $x$-direction

Figure 4-2: Small channelized system. Variational multiscale solution on a $2 \times 2$ coarse grid.

The fine-scale finite volume solution predicts an overall flow rate across the domain of 0.2064 , and the VMS solution a flow rate of 0.2422 (a $17 \%$ error). For comparison, the flow rate computed with the finite volume method on a refined grid of $16 \times$ 16 elements is 0.2477 . This represents a $17 \%$ difference with respect to the flow rate computed on a $4 \times 4$ grid on which the permeability is defined. In this particular case, the error between VMS and fine-scale finite volume solutions is thus of the same order as the error between finite volume solutions on the fine-scale and on the refined grid. In the examples that follow, the VMS solution on a number of coarse grids is compared only with the finite volume solution on the original fine grid in which the permeability is defined.

\subsection{Quarter five-spot simulations}

Here we show results for corner-to-corner flow in a 2D square geometry, a configuration known as a quarter of a five-spot pattern. Injector and producer are located in diagonally opposite vertices of the grid (Figure 4-3), and all boundaries are no-flow boundaries. Wells at the corners of the domain are modeled as source/sink terms over the fine-scale corner grid block. We used a fine grid of $64 \times 64$ elements. 


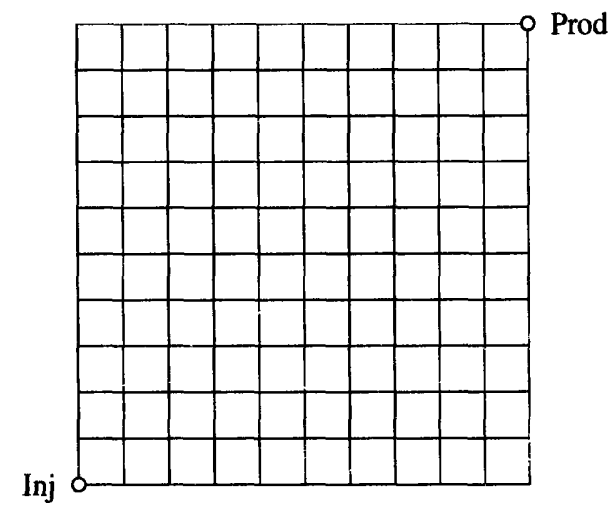

Figure 4-3: Schematic of the quarter five-spot problem.

The objective of this section is to study the performance of the proposed VMS formulation for:

- Different heterogeneous permeability fields: smoothly-varying and channelized.

- A variety of coarse meshes: from 2 - to 16 -fold upgridding $(N=32 \times 32$ to $4 \times 4$, respectively).

- Various choices of the well length scale: well region ranging from $2 \times 2$ to $16 \times 16$ fine blocks ( $L_{w}=1 / 32$ to $1 / 4$, respectively).

\subsubsection{Isotropic correlation structure}

In this heterogeneous structure, the permeability is isotropic and log-normally distributed with dimensionless correlation length equal to 0.1 in each direction, and $\sigma_{\log k}^{2} \approx 1.0$. The permeability field is shown in Figure 4-4.

In Figure 4-5 we show the finite volume solution (fine-scale pressure, $x$-flux, $y$-flux) computed on the fine grid, and in Figure 4-6 we show the solution with the VMSFE method on a coarse grid $4 \times 4$ elements (a $16 \times 16$ upgridding), with a length scale $N_{l}=1 / 4$. The multiscale solution captures the behavior of the fluid on the fine scale, although the pressure displays slight oscillations due to the large upgridding.

In Figure 4-7 we plot the velocity and pressure errors for different coarse discretizations and well length scales used to compute the fine well contributions. As 
expected, the error decreases with increasing number of coarse cells. In this case, the multiscale solutions are fairly independent of the well length scale although larger support for the well basis function results in slightly smaller error.

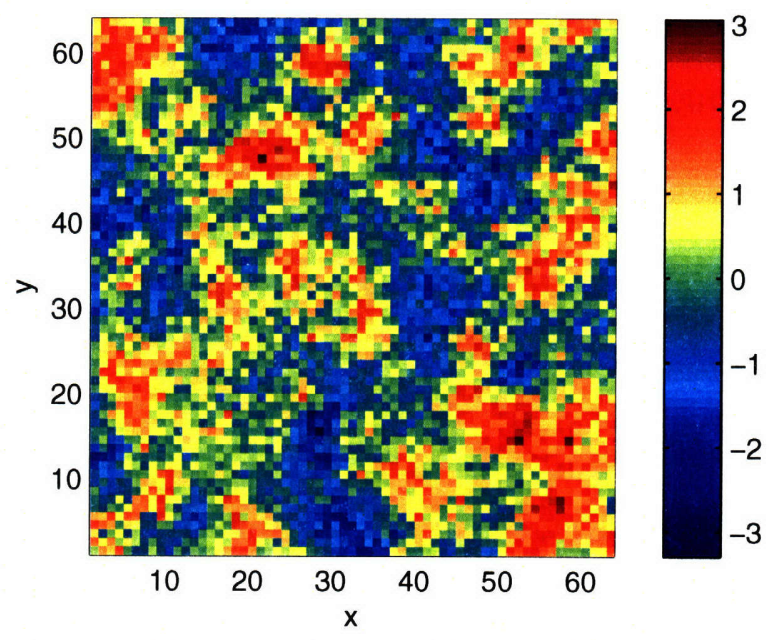

Figure 4-4: Log-permeability field for the isotropic correlation structure.

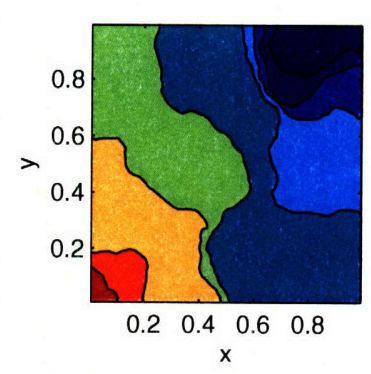

(a) pressure
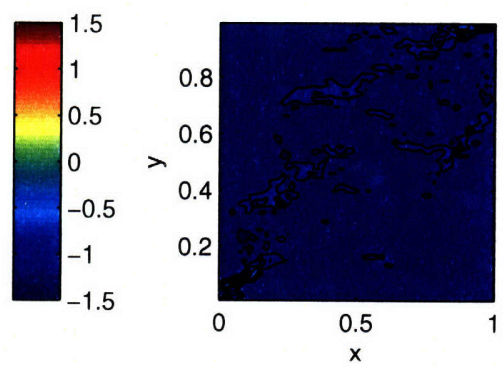

(b) $x$-flux
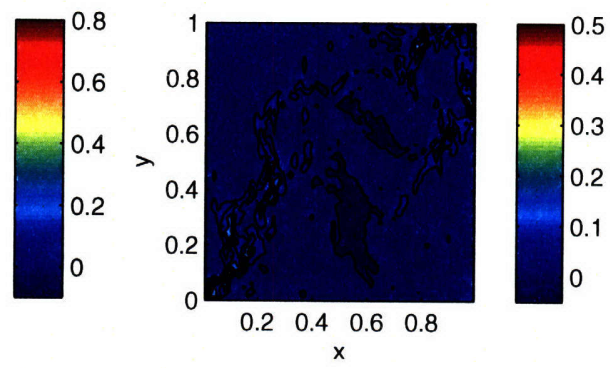

(c) $y$-flux

Figure 4-5: Isotropic correlation structure. Finite volume solution on the fine $64 \times$ 64 grid. 


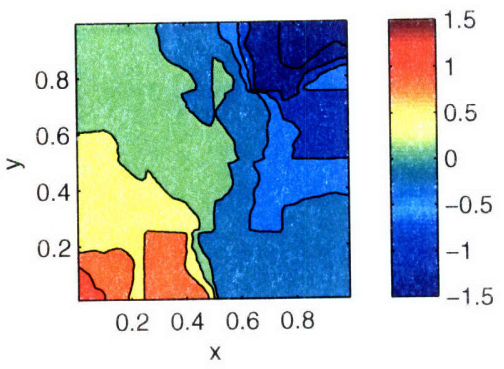

(a) pressure

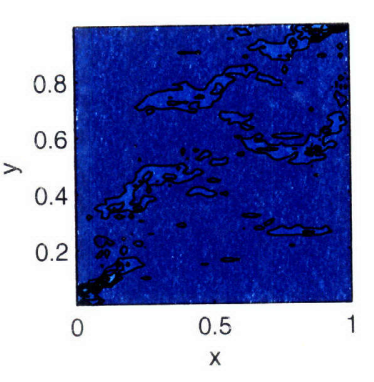

(b) $x$-flux
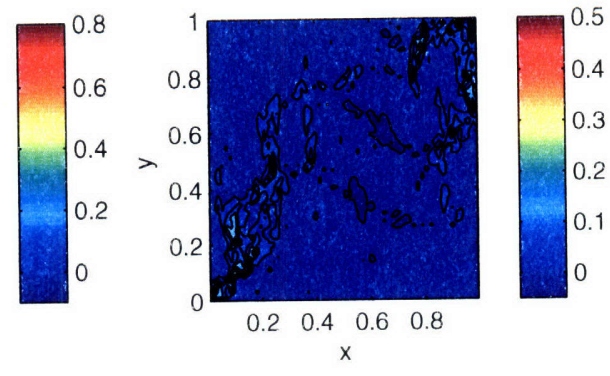

(c) $y$-flux

Figure 4-6: Isotropic correlation structure. Variational multiscale solution on a $4 \times$ 4 grid $\left(16 \times 16\right.$ upgridding) with well length scale $N_{l}=1 / 4$.
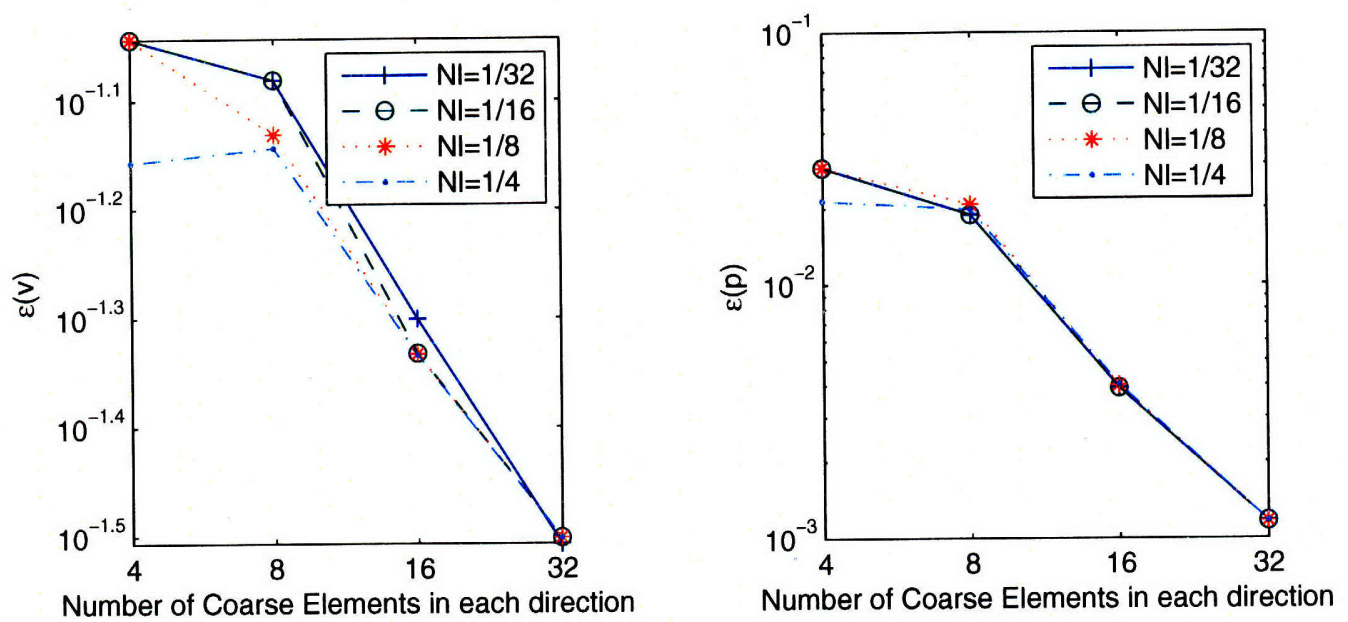

Figure 4-7: Velocity (left) and pressure (right) errors for the isotropic correlation scenario.

\subsubsection{System with vertical channels}

Here we present results for a highly heterogeneous system (the permeability varies over 8 orders of magnitude) in which the heterogeneity is dominated by a large correlation length (about 1.0) in the $y$-direction. The correlation length in the $x$-direction is around 0.1, as before. The log-permeability map is shown in Figure 4-8. 


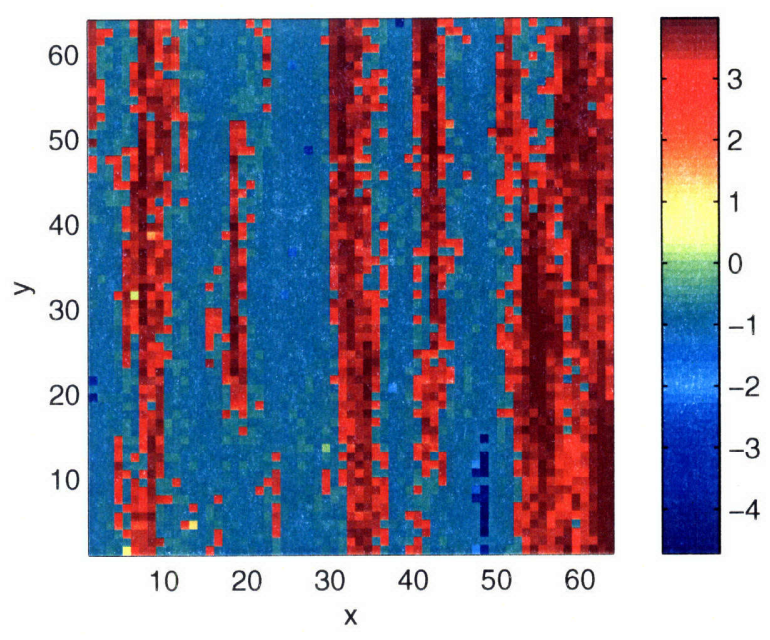

Figure 4-8: Log-permeability field for the structure dominated by vertical channels.

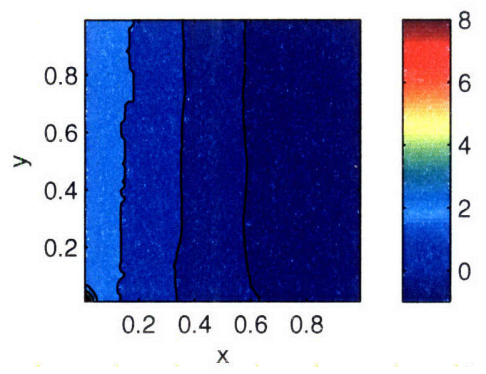

(a) pressure

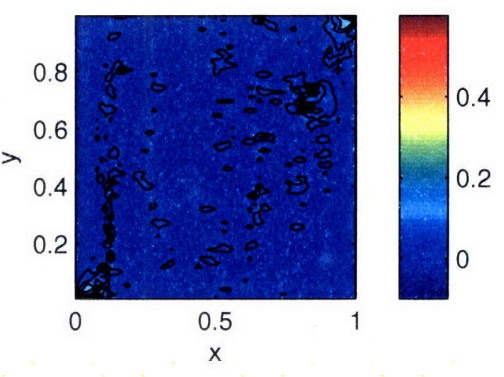

(b) $x$-flux

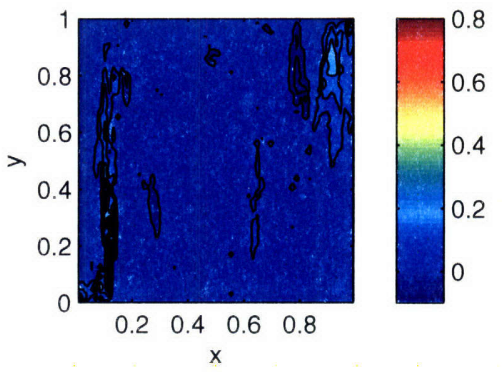

(c) $y$-flux

Figure 4-9: Isotropic correlation structure. Finite volume solution on the fine $64 \times$ 64 grid. 


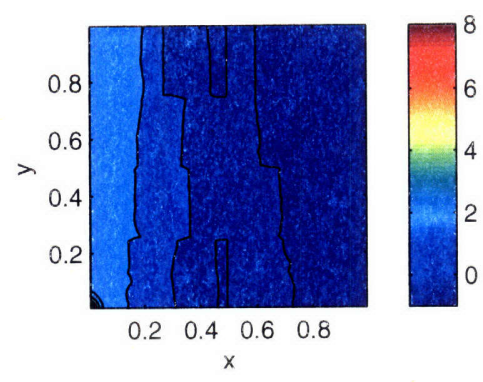

(a) pressure

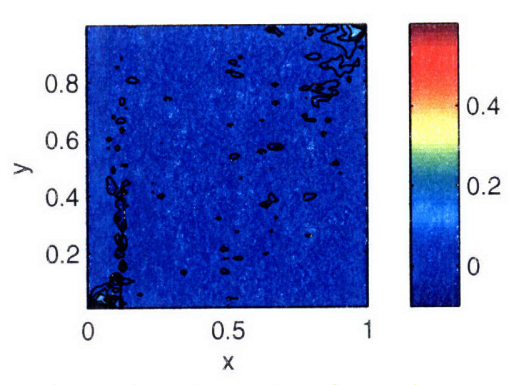

(b) $x$-flux

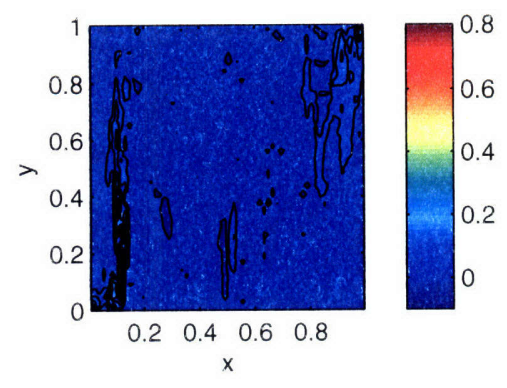

(c) $y$-flux

Figure 4-10: Isotropic correlation structure. Variational multiscale solution on a $4 \times 4$ grid $\left(16 \times 16\right.$ upgridding) with well length scale $N_{l}=1 / 4$.
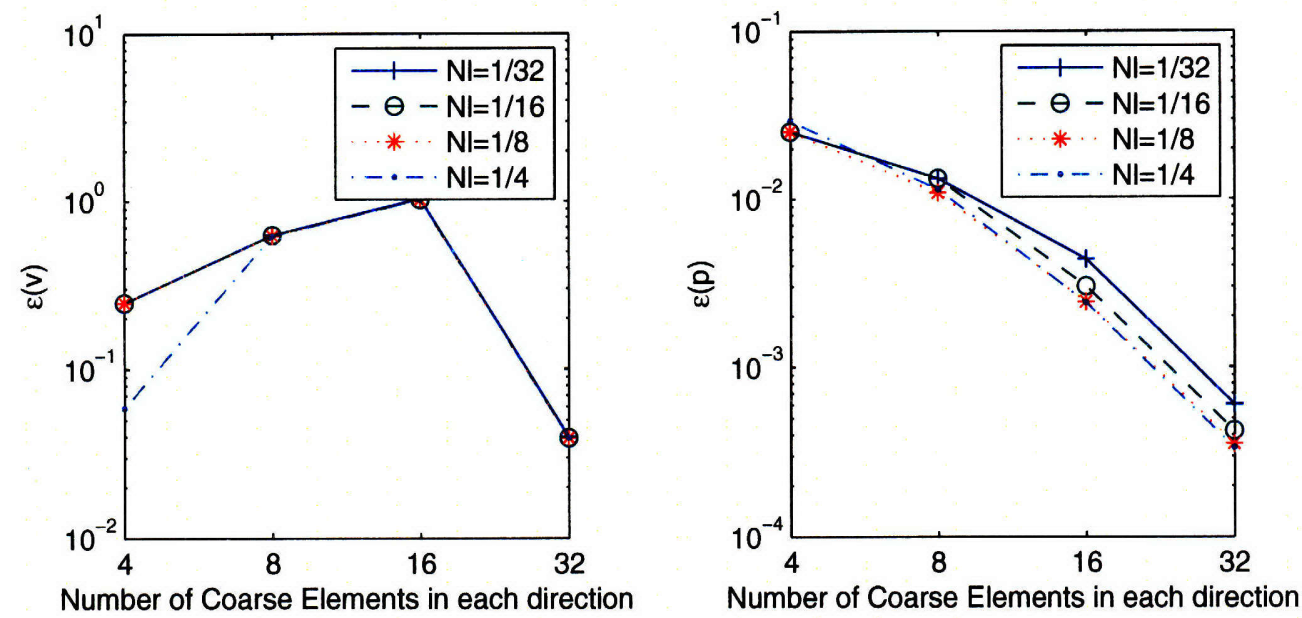

Figure 4-11: Velocity (left) and pressure (right) errors for the scenario with vertical channels.

The velocity and pressure fields obtained with the finite volume method on the $64 \times 64$ grid are shown in Figure 4-9. The solution obtained on a coarse grid $4 \times 4$ elements (a $16 \times 16$ upgridding), with a length scale $N_{l}=1 / 4$ are shown in Figure 4-10. The multiscale solution is accurate, although the pressure field lacks some precision at the interface between elements. The velocity and pressure errors are shown in Figure 4-11. The pressure error decreases monotonically as the coarse grid is refined, and it decreases also (albeit slightly) with increasing well length scale. The velocity error displays a somewhat different behavior: the error is larger for a coarse grid of $8 \times 8$ or $16 \times 16$ elements than for a coarse grid of $4 \times 4$ elements. The reason 
is that due to the drastic changes in permeability in the $x$-direction, the VMSMFE exhibits a mild resonance effect for coarse discretizations of the same length scale as the $x$-correlation length.

\subsubsection{System with diagonal channels}

Similar observations apply for a heterogeneous permeability field with large correlation length in the diagonal direction (Figure 4-12). The pressure is captured with precision but displays oscillations at the interface between coarse elements, the velocity is accurate (Figure 4-13 and Figure 4-14). The pressure error decreases monotonically with increasing refinement of the coarse grid and well length scale. The velocity error, on the other hand, is small but does not exhibit a clear trend with respect to the coarse discretization (Figure 4-15).

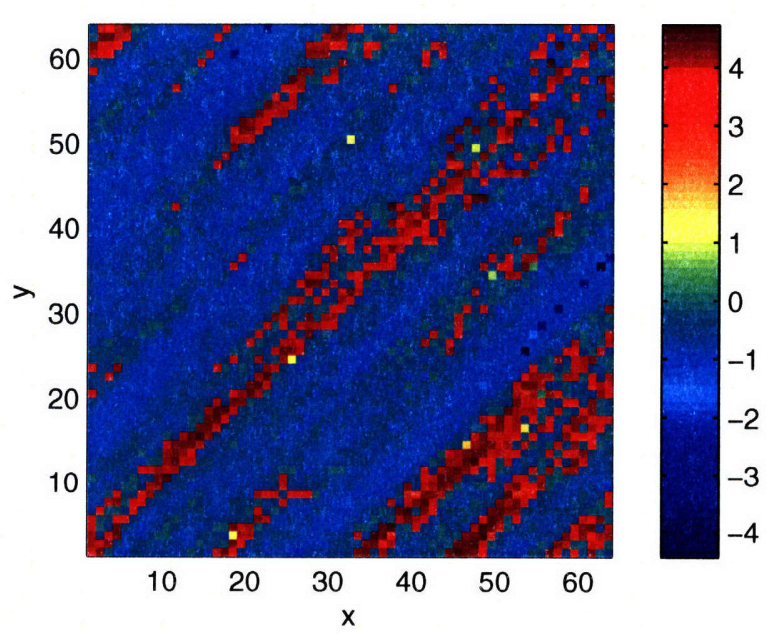

Figure 4-12: Log-permeability field for the structure dominated by diagonal channels. 


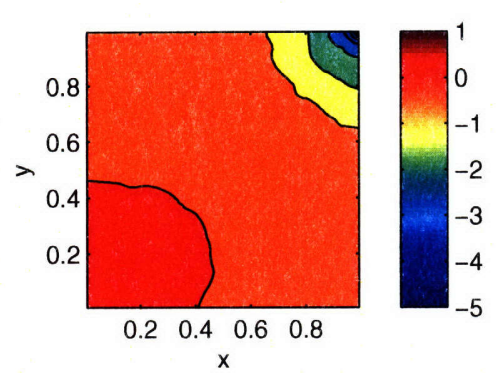

(a) pressure

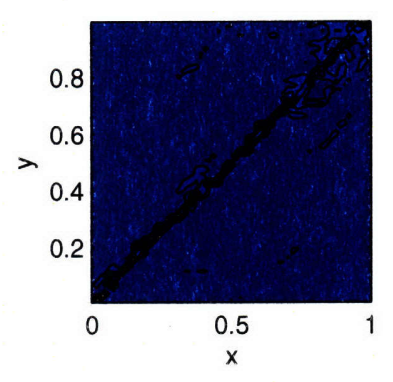

(b) $x$-flux
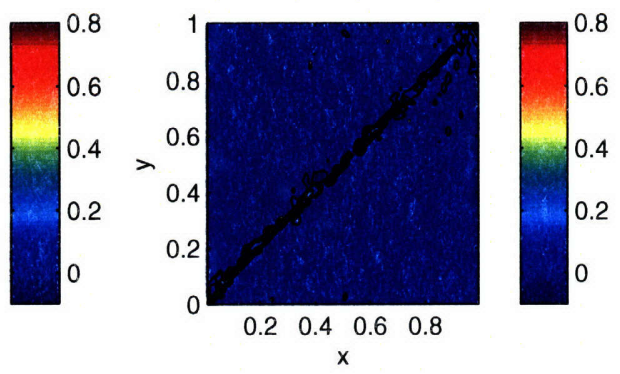

(c) $y$-flux

Figure 4-13: Structure dominated by diagonal channels. Finite volume solution on the fine $64 \times 64$ grid.

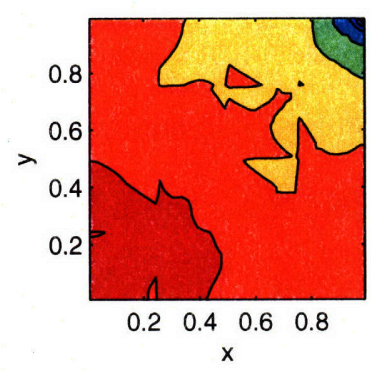

(a) pressure

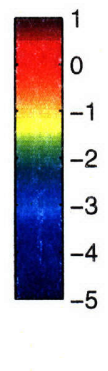

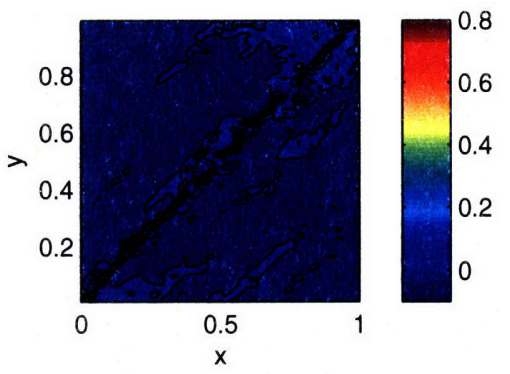

(b) $x$-flux

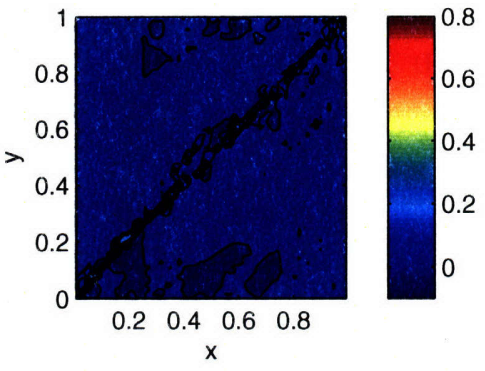

(c) $y$-flux

Figure 4-14: Structure dominated by diagonal channels. Variational multiscale solution on a $4 \times 4$ grid $(16 \times 16$ upgridding $)$ with well length scale $N_{l}=1 / 4$.
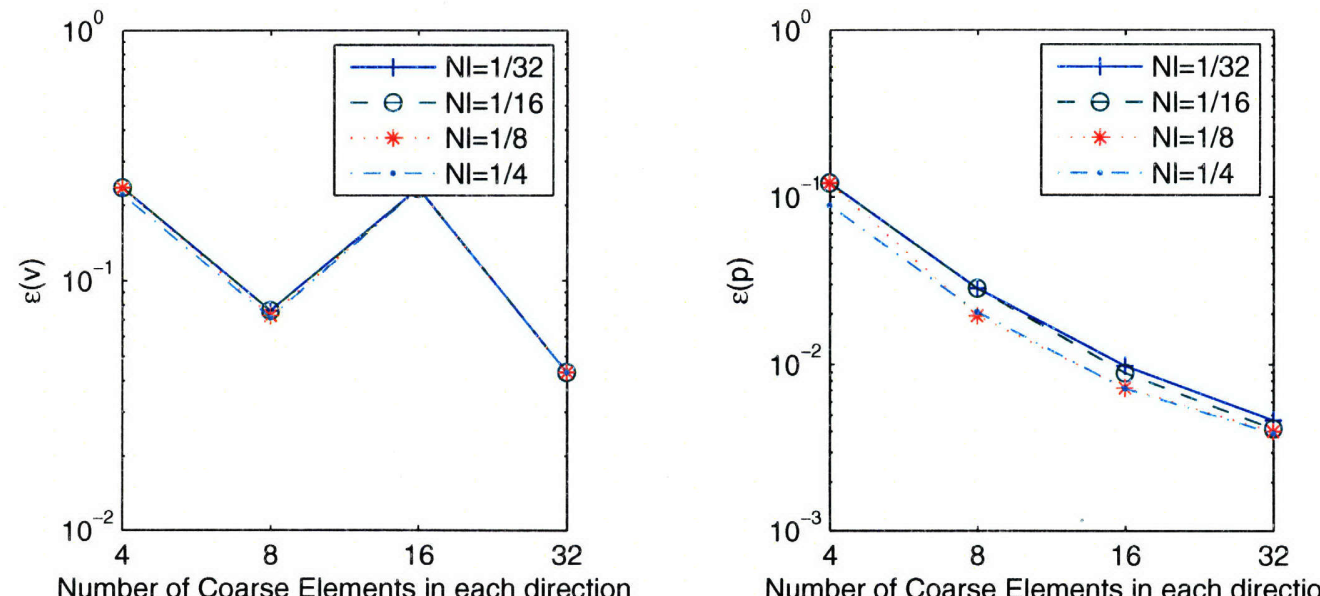

Number of Coarse Elements in each direction

Figure 4-15: Velocity (left) and pressure (right) errors for the scenario with diagonal channels. 


\subsection{Study of anisotropy}

It has been reported recently $[28,30]$ that some multiscale methods have difficulty in producing high quality solutions in the presence of permeability anisotropy or large aspect ratios of the grid (both cases are common in reservoir models). Here, we demonstrate the ability of our proposed method to cope with these scenarios. We consider diagonal permeability tensors

$$
\mathbf{k}=\left(\begin{array}{cc}
k_{x x} & 0 \\
0 & k_{y y}
\end{array}\right),
$$

where $\alpha=k_{y y} / k_{x x}$ is the anisotropy ratio. To fully explore the effect of anisotropy, we used values of $\alpha$ ranging from 0.01 to 100 (the same value of $\alpha$ is used for all gridblocks in any given simulation). In order to isolate the effect of anisotropy, but avoid the physically unrealistic case of homogeneous media, we perturbed the permeability field with

$$
k_{x x}=1+\delta
$$

where $\delta$ is a random number, uniformly distributed and of magnitude bounded by $|\delta| \leq 0.01$. The actual log-permeability $\left(k_{x x}\right)$ field defined on a $64 \times 64$ fine grid is shown in Figure 4-16. 


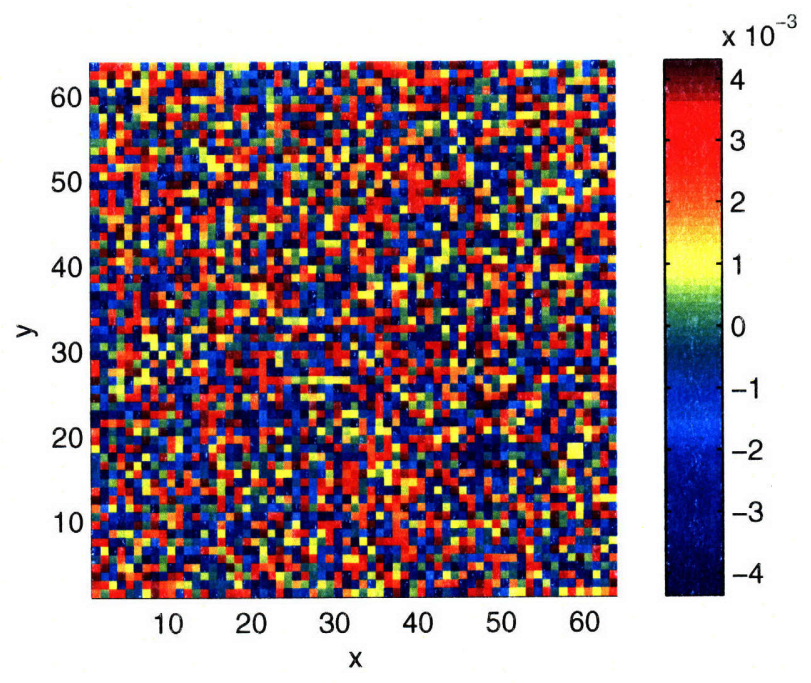

Figure 4-16: Log-permeability $\left(k_{x x}\right)$ field for the study of anisotropy.

The flow scenario is the same as the one described in the previous section: no-flow boundaries, with an injection well at the bottom-left corner and a producer at the top-right corner of the square domain. This is in fact a challenging test case that leads to the formation of boundary layers. The fine grid is a $64 \times 64$ grid and we make use of several coarse meshes with unit aspect ratio: 4, 8, 16 and 32. As an illustration of the good behavior of the method, we show in Figure 4-17 (resp. Figure 4-18) the pressure field computed with a finite volume method on the fine grid and the VMSMFE method for a value of the anisotropy ratio of $\alpha=0.01$ (resp. $\alpha=0.1$ ). The multiscale solution was computed on a $8 \times 8$ coarse grid, and with a well length scale $N_{l}=1 / 4$. For comparison, we plot in Figure 4-19 the pressure field in the isotropic case. 

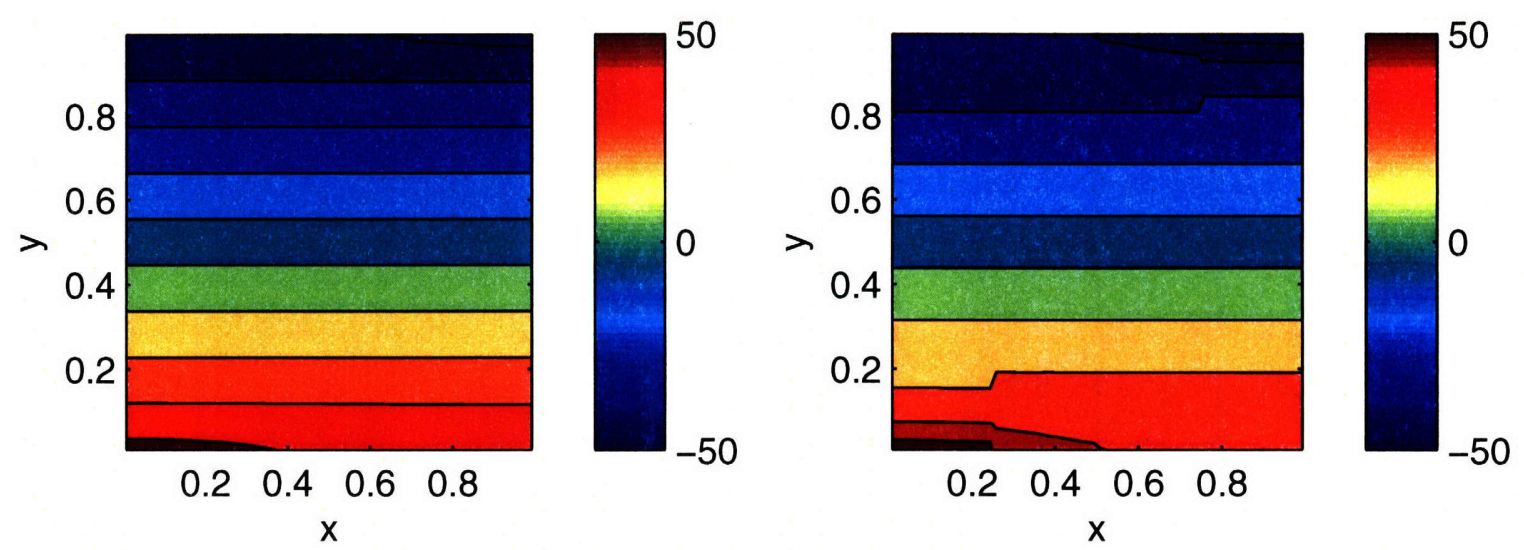

Figure 4-17: Pressure field for the anisotropic permeability field $(\alpha=0.01)$. Left: fine-scale finite volume solution. Right: variational multiscale solution on a $8 \times 8$ grid ( $8 \times 8$ upgridding) .
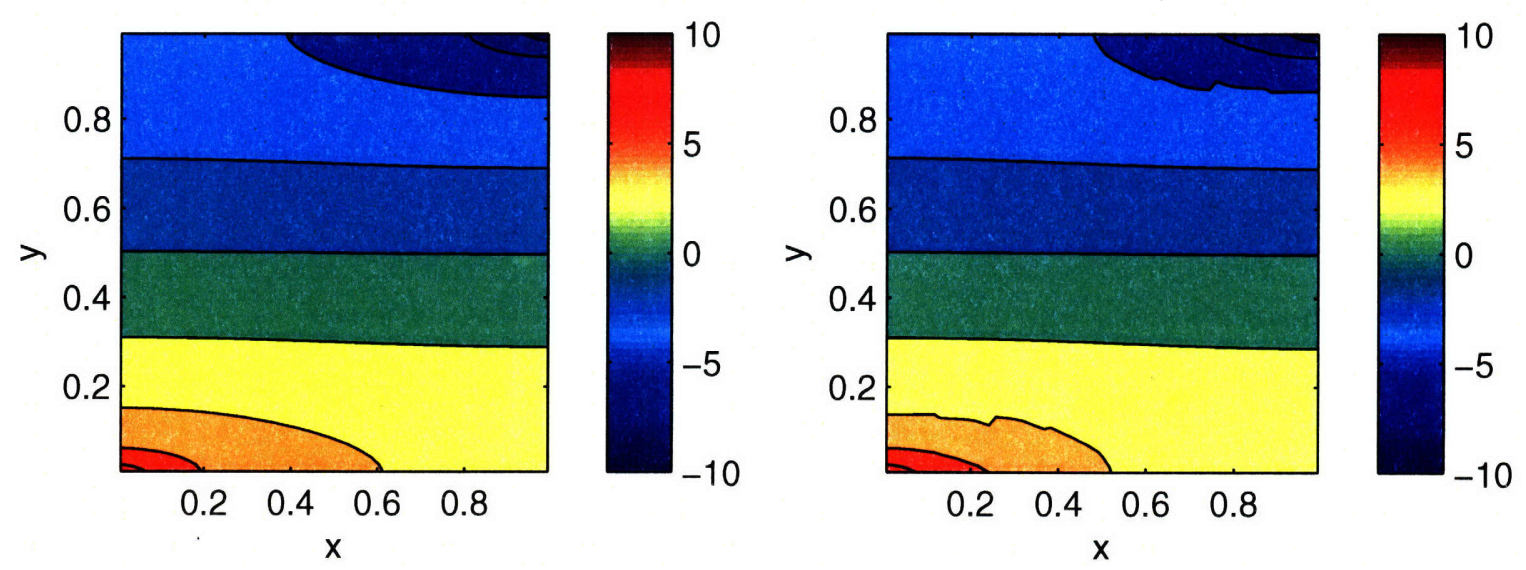

Figure 4-18: Pressure field for the anisotropic permeability field $(\alpha=0.1)$. Left: fine-scale finite volume solution. Right: variational multiscale solution on a $8 \times 8$ grid $(8 \times 8$ upgridding $)$. 

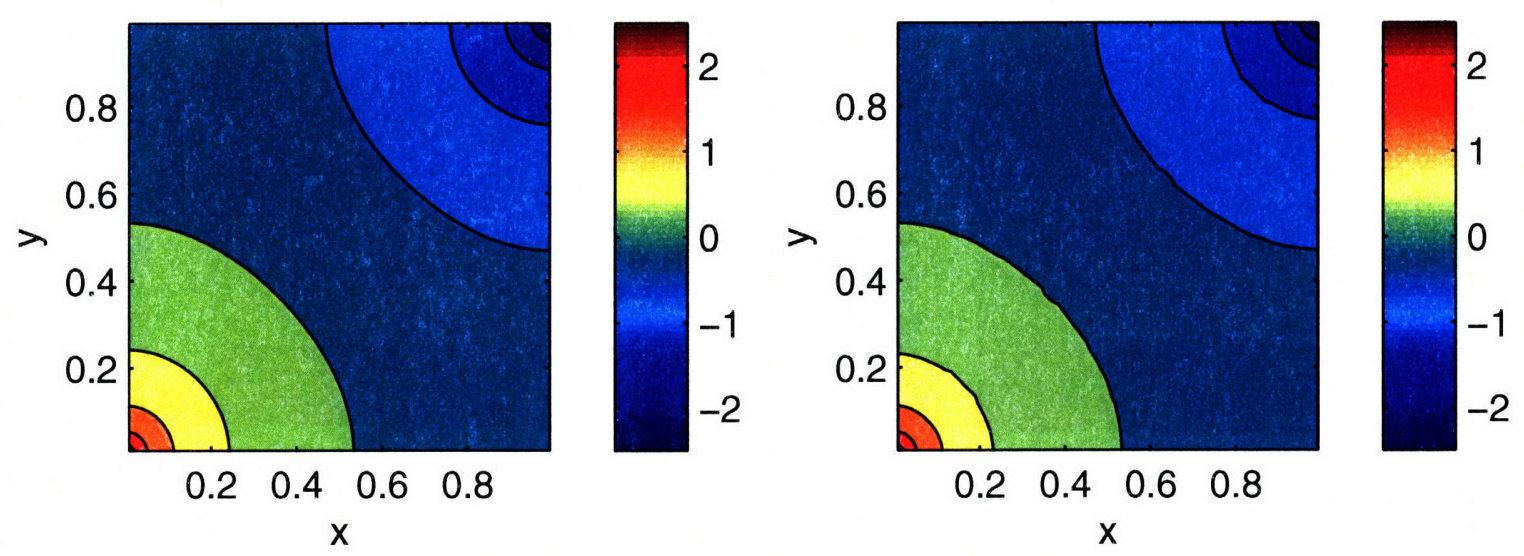

Figure 4-19: Pressure field for the isotropic permeability field $(\alpha=1)$. Left: finescale finite volume solution. Right: variational multiscale solution on a $8 \times 8$ grid $(8 \times 8$ upgridding $)$.

Figure 4-20 shows the velocity and pressure errors for different anisotropy ratios $\alpha$ and different choices of the coarse grid. The well length scale is set at a value $N_{l}=1 / 4$ for all computations. The results reveal the very robust behavior of the proposed VMSMFE method with respect to anisotropy. For $\alpha=1$ (isotropic), the method yields a very accurate solution for all coarse discretizations: $\varepsilon(\boldsymbol{u}) \approx 10^{-2}$ for all coarse grids, and $\varepsilon(p)$ decreases monotonically as the coarse grid is refined. Another indication of the robustness of the method is that the results for $\alpha=10$ (resp. $\alpha=$ 100) are virtually identical to those for $\alpha=0.1$ (resp. $\alpha=0.01$ ). Of course, increasing levels of anisotropy $(\alpha=10,100)$ lead to larger velocity and pressure errors. However, the errors are still small, and they decrease with refinement of the coarse grid. 

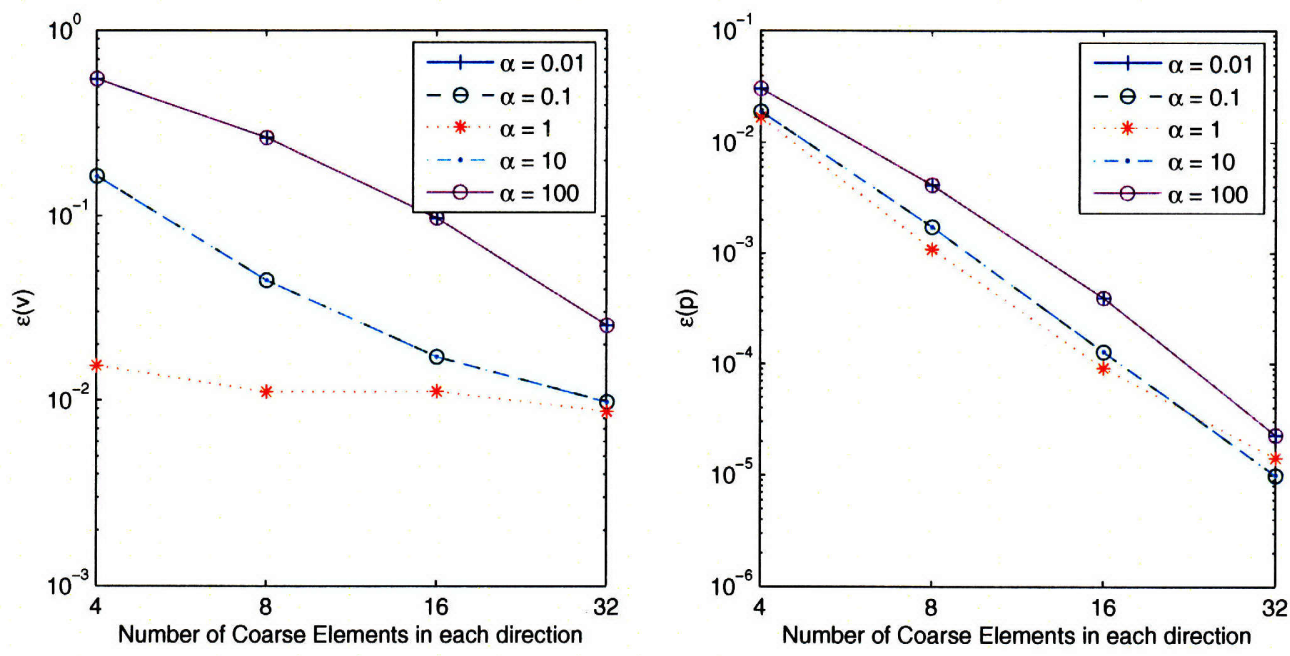

Figure 4-20: Velocity (left) and pressure (right) errors for the anisotropic permeability field.

\subsection{Simulations in realistic $2 \mathrm{D}$ reservoirs}

\subsubsection{Highly heterogeneous, smoothly-varying permeability}

This test case is a two-dimensional problem with a highly heterogeneous isotropic permeability. The permeability field, shown in Figure 4-21(a), has large (but smooth) variations of 6 orders of magnitude. It is taken from the top layer of the 10th SPE comparative solution project [17]. The fine grid on which the permeability is defined consists of $60 \times 220$ gridblocks. All boundaries are no-flow boundaries. Flow is driven by an injection well at the center of the domain and four production wells, one at each corner (see Figure 4-21(a)). Production wells are modeled as sinks over one fine gridblock - the corner gridblock. To respect symmetry, the production well is modeled as a source over four gridblocks on the fine grid. The location of fine-scale sources and sinks is independent of the choice of the coarse grid. Therefore, the wells may be placed at the center or at the boundary of a coarse element, depending on the coarse discretization.

The finite volume solution computed on the fine grid is shown in Figure 4-21. Most of the pressure drop occurs at the low permeability region $20<y<40$. The 
contour plots of $x$ - and $y$-flux clearly indicate flow focusing along the more permeable regions, bypassing the low-permeability areas. The velocity field displays, however, significant small-scale structure in response to the spatial permeability variations.

In Figure 4-22 we show the solution (fine-scale pressure, $x$-flux and $y$-flux) obtained with the VMSMFE method on a coarse grid of $6 \times 22$ elements (each containing $10 \times$ 10 fine blocks) and a well length scale of $N_{l}=1 / 4$. Despite the severe heterogeneity and the rather aggressive 100-fold upgridding, the multiscale solution is remarkably accurate. The velocity error is $\varepsilon(\boldsymbol{u})=0.185$. Moreover, both the large and small scale flow patterns are captured with very high fidelity. It should be noted, however, that even though the average (coarse) pressure field is accurate, the reconstructed (fine) pressure presents slight oscillations at the interfaces between coarse elements.

In Figure 4-23 we show the velocity and pressure error of the variational multiscale method for a fixed length scale $N_{l}=1 / 4$ as a function of the coarse discretization into $N_{x} \times N_{y}$ elements, for all combinations of $N_{x}=\{6,10,30\}$ and $N_{y}=\{11,22,55,110\}$. In this way, we test the robustness of the method with respect to not only coarseness of the (coarse) grid, but also to (coarse) grid aspect ratio. We make the following observations:

- The method displays very good behavior even for aggressive upgridding. As expected, the error is smaller for discretizations with aspect ratio close to unity $(6 \times 22,10 \times 55$ and $30 \times 110$ grids $)$, and it decreases with refinement of the coarse grid.

- The pressure error is very small, between $10^{-3}$ and $10^{-2}$ in most cases. Pressure errors are larger, however, for grids with $N_{y}=11$. These grids have coarse elements with 20 fine gridblocks in the $y$-direction-approximately the extent of the low-permeability zone.

- The velocity error is larger for grids with a large aspect ratio (notably, for the $6 \times 110$ coarse grid). 


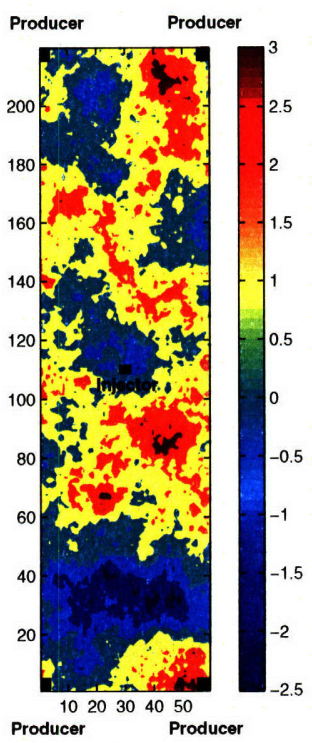

(a) $\log$ perm.

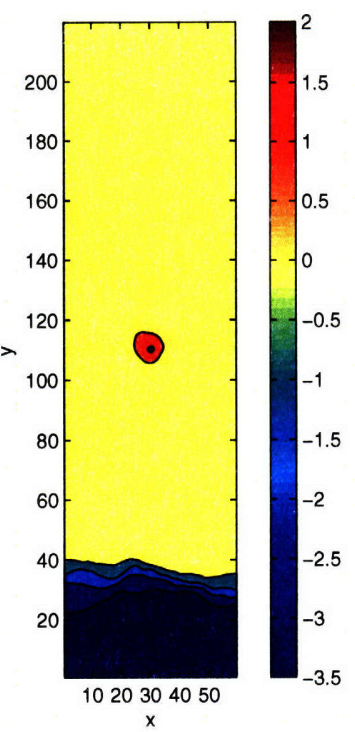

(b) pressure

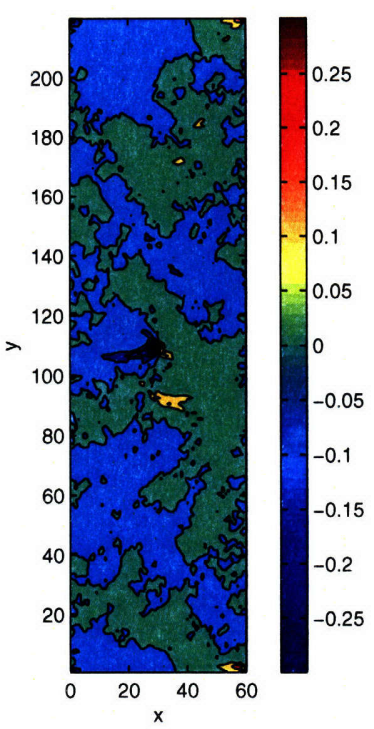

(c) $x$-flux

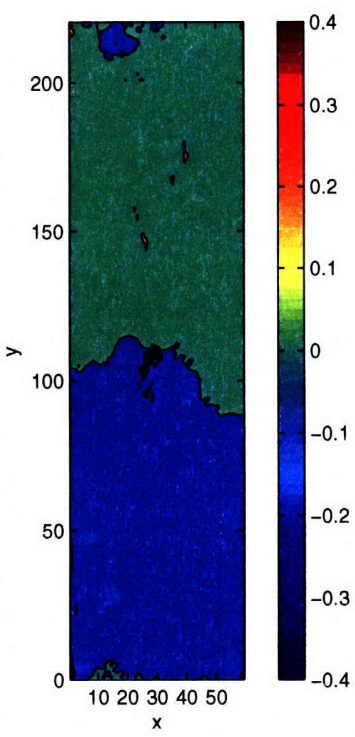

(d) $y$-flux

Figure 4-21: Smooth permeability field. Finite volume solution on the fine $60 \times$ 220 grid.

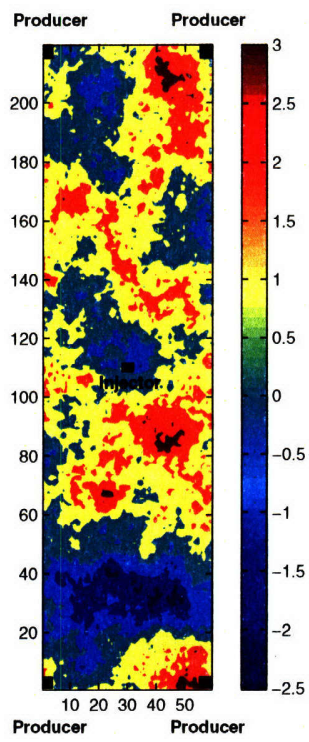

(a) $\log$ perm.

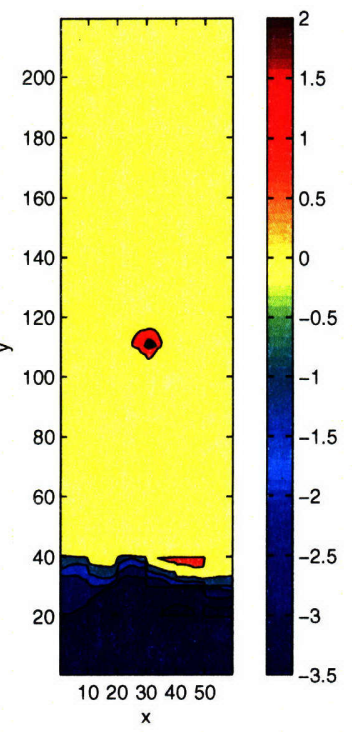

(b) pressure

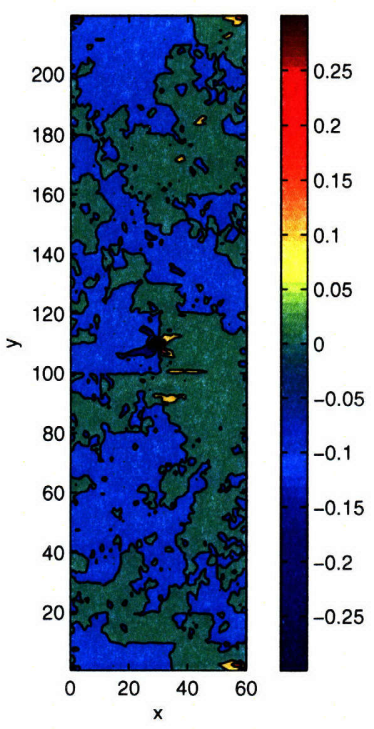

(c) $x$-flux

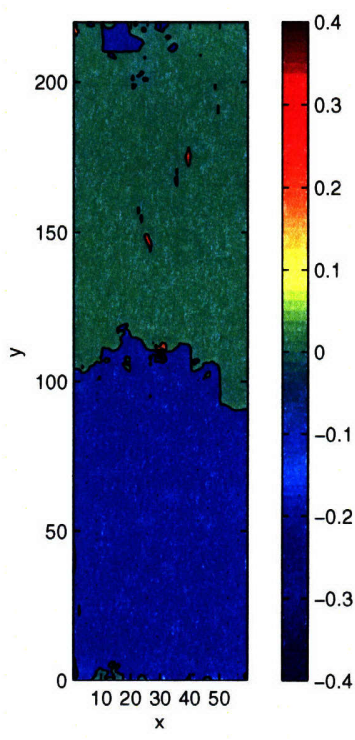

(d) $y$-flux

Figure 4-22: Smooth permeability field. Variational multiscale solution on a $6 \times 22$ grid $\left(10 \times 10\right.$ upgridding) with well length scale $N_{l}=1 / 4$. 

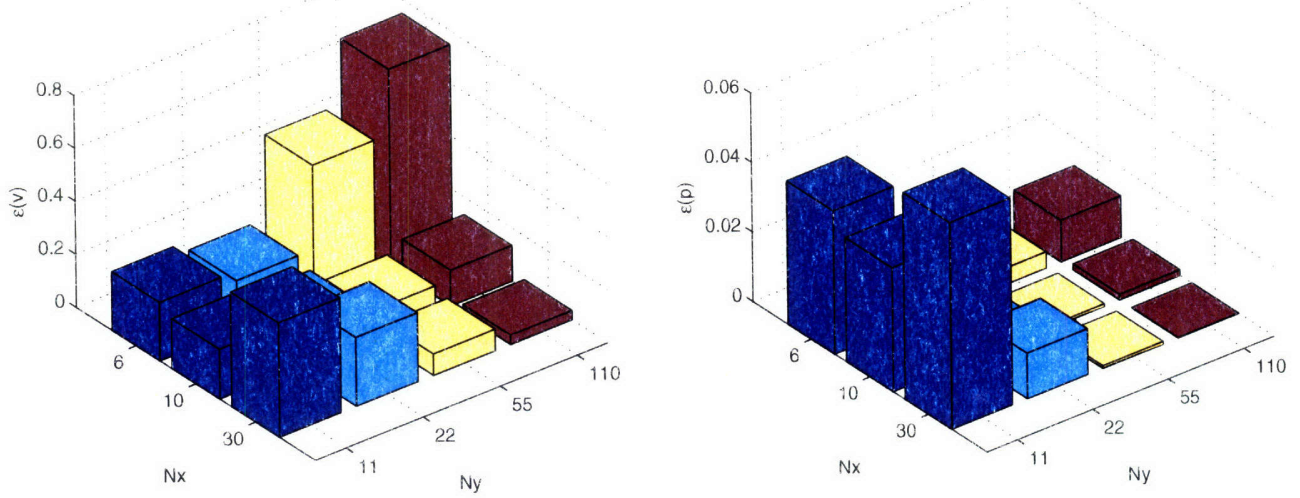

Figure 4-23: Smooth permeability field. Velocity and pressure errors for different coarse discretizations.

\subsubsection{Highly heterogeneous, channelized permeability}

This test case is a challenging two-dimensional problem with a highly heterogeneous, but now rough (channelized) permeability field (see Figure 4-24(a)). It is a synthetic model of a fluvial reservoir, taken from Layer 59 of the 10th SPE comparative solution project. The fine grid, boundary conditions and well locations are the same as in the previous example.

In Figure 4-24 we plot the pressure and fluxes from a fine-grid finite volume solution. The most salient feature is the pronounced focusing of flow along the highpermeability channels, leading to very high velocities (in both $x$ - and $y$-directions) over very narrow regions of the domain. This is also true near the injection and production wells.

The variational multiscale solution is shown in Figure 4-25. It was computed, as before, on a coarse grid of $6 \times 22$ elements, each containing $10 \times 10$ fine blocks, using a well length scale of $N_{l}=1 / 4$. The method is able to capture the high-velocity regions very accurately, even though they have a long range in the streamwise direction and a very short range in the spanwise direction. The global flow pattern is reproduced accurately as well. This is evidenced by the small mean velocity error of $\varepsilon(\boldsymbol{u})=0.330$. Clearly, an essential ingredient of the proposed method is its ability to account for subgrid variability at the element interfaces. Once again, the multiscale pressure 
solution agrees well with the fine-grid finite volume solution on an average sense, but presents mild discrepancies after the fine-scale reconstruction step, including spurious local maxima at coarse element interfaces.

The mean velocity and pressure errors of the variational multiscale method for a fixed well length scale $N_{l}=1 / 4$ and for various coarse grids are shown in Figure 4-26. As in the previous example, the bar plot displays a valley (minima) for discretizations with grid aspect ratio close to unity $(6 \times 22,10 \times 55$ and $30 \times 110)$, and hills (maxima) for discretizations with large aspect ratios $(30 \times 11$ and $6 \times 110)$.

Given the large values of the velocity error $\varepsilon(\boldsymbol{u})$, we investigate the applicability of the variational multiscale method for the accurate simulation of transport problems. We simulate the transport of a passive tracer, described by the advection equation, using a single-point upstream discretization, and an explicit forward Euler time stepping [8]. The velocity field is fixed from the solution to the pressure equation. We use the reconstructed fine-scale fluxes obtained with the VMS method on various coarse grids. The computed concentration field on a number of different coarse grids is shown in Figure 4-27. The concentration fields are of very high quality, even for the coarse grids on which the fluxes display large errors (that is, the $30 \times 22$ and $30 \times 55$ grids) . 


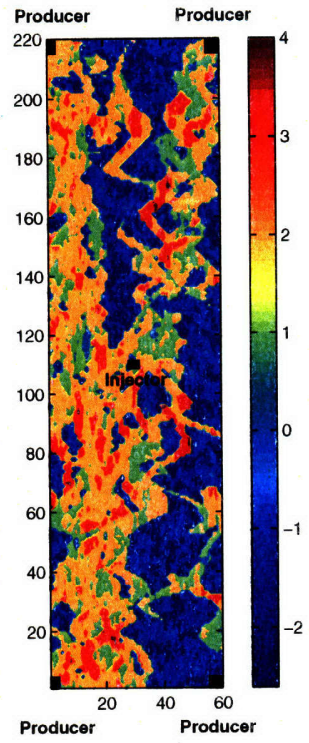

(a) $\log$ perm.

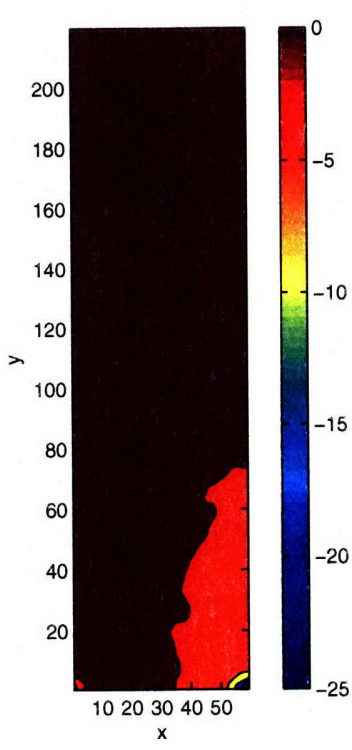

(b) pressure

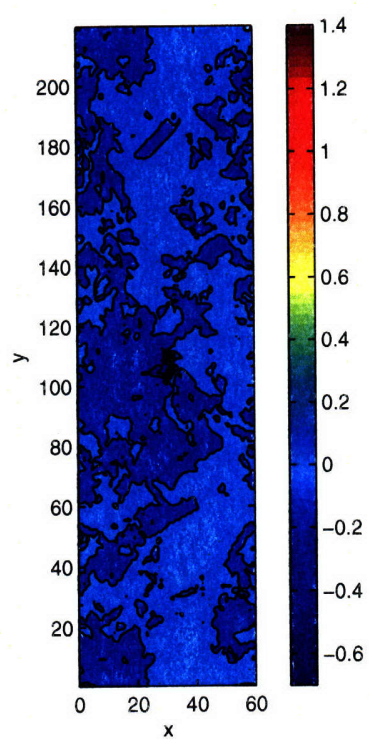

(c) $x$-flux

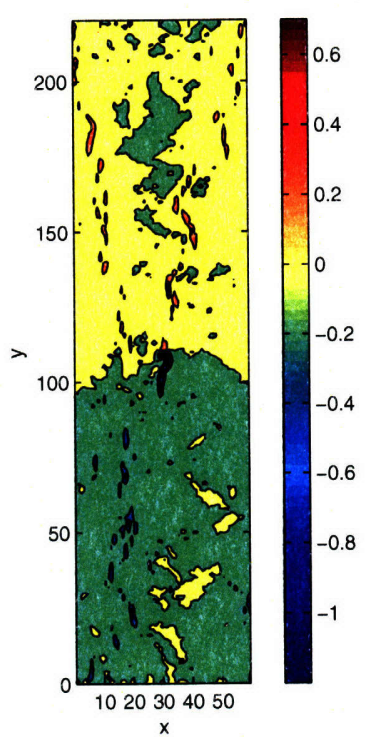

(d) $y$-flux

Figure 4-24: Channelized permeability field. Finite volume solution on the fine $60 \times$ 220 grid.

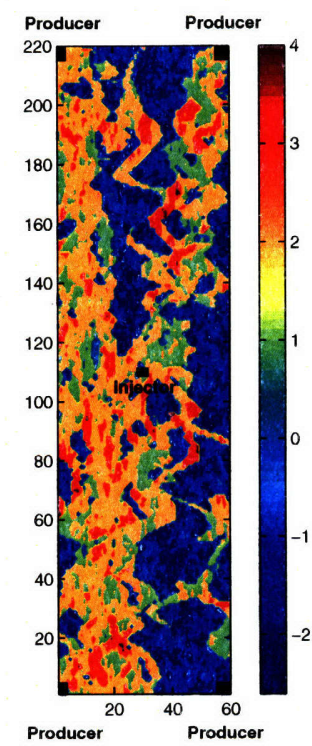

(a) $\log$ perm.

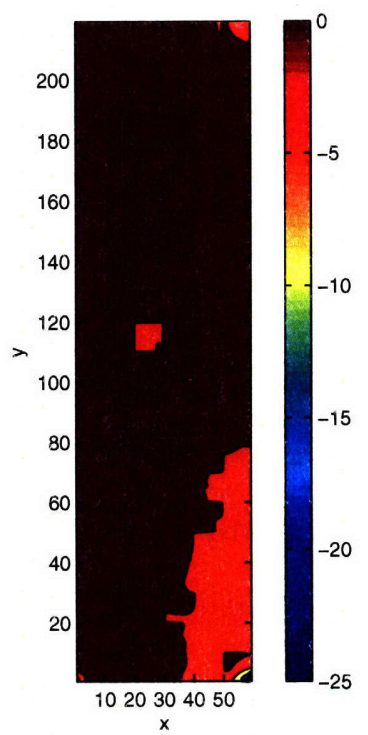

(b) pressure

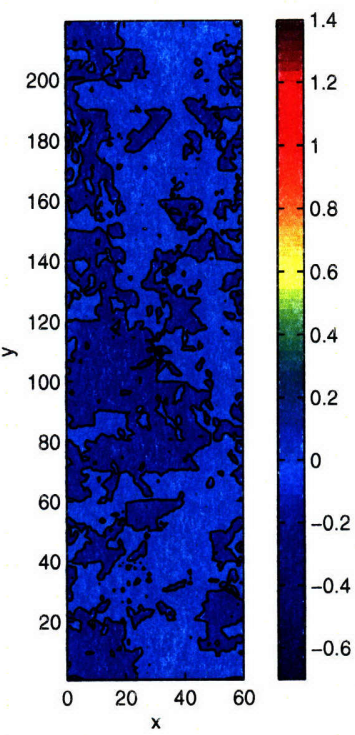

(c) $x$-flux

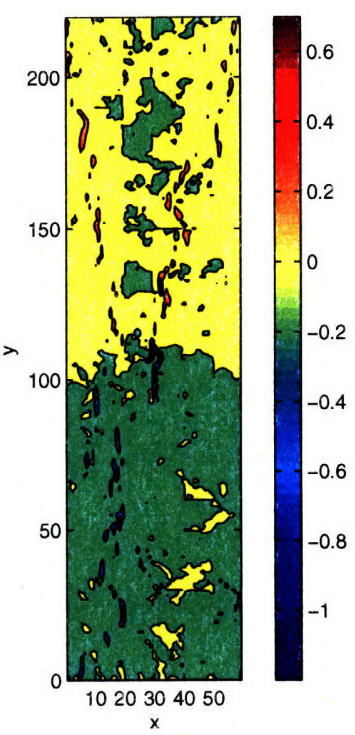

(d) $y$-flux

Figure 4-25: Channelized permeability field. Variational multiscale solution on a $6 \times 22$ grid $(10 \times 10$ upgridding $)$ with well length scale $N_{l}=1 / 4$. 

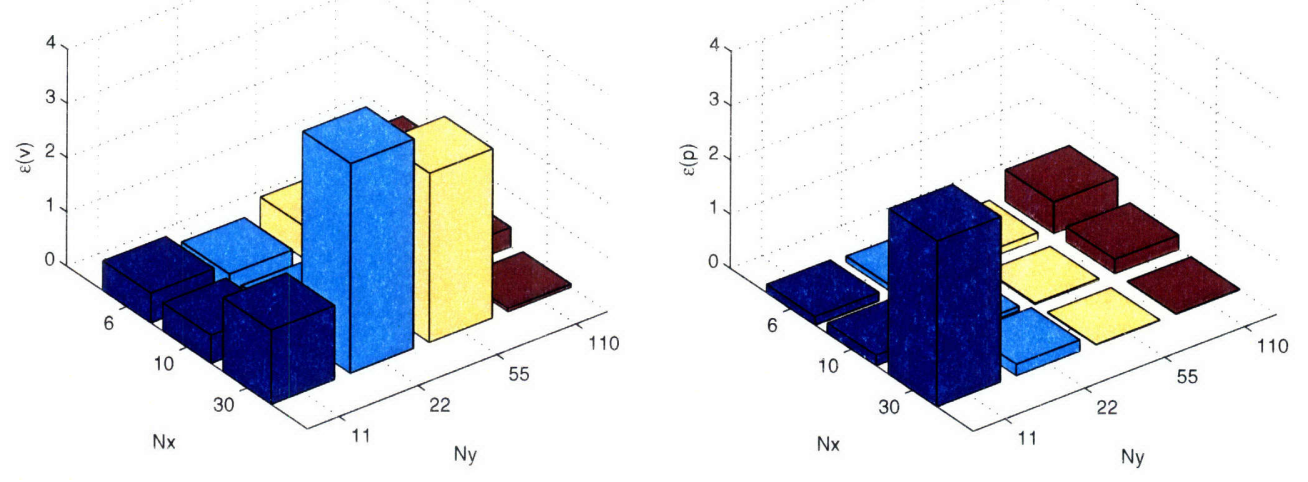

Figure 4-26: Channelized permeability field. Velocity and pressure errors for different coarse discretizations.

The variational multiscale method is also able to capture the behavior of the breakthrough curves of the passive tracer at the production wells. As shown in Figure 4-28, the breakthrough curves computed with the $6 \times 22$ and $30 \times 55$ grids show very mild inaccuracies. The results for the $30 \times 22$ grid are less accurate, but still far better than the velocity error of $\varepsilon(\boldsymbol{u}) \approx 4$ would suggest. These results show that the VMS method is applicable for accurate simulation of transport problems, and that the measure given by the global velocity error $\varepsilon(\boldsymbol{u})$ may be overly pessimistic. 


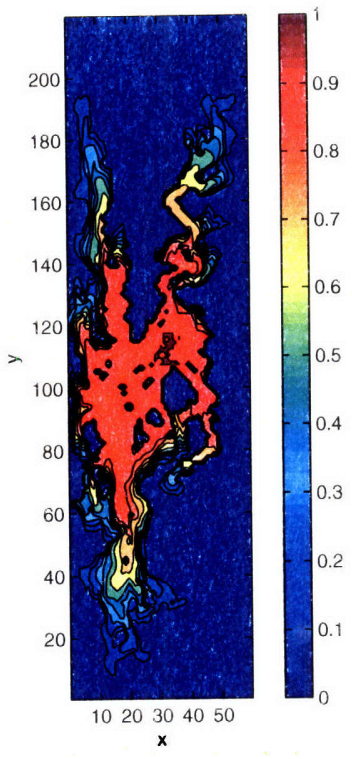

(a)

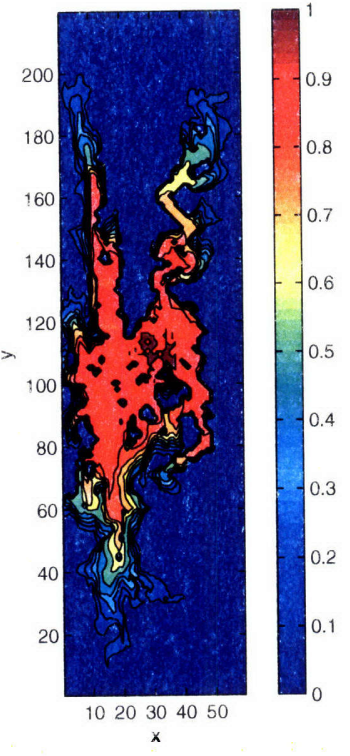

(b)

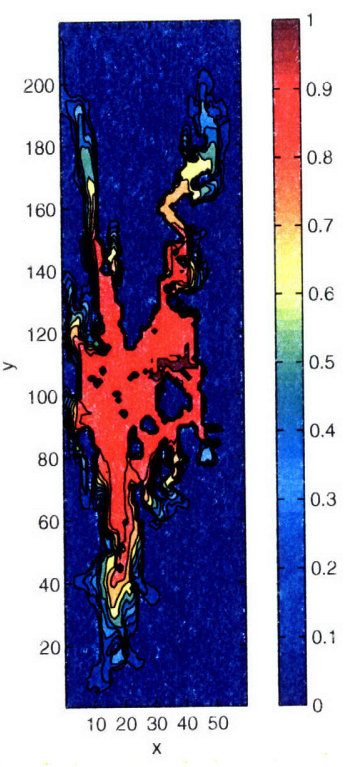

(c)

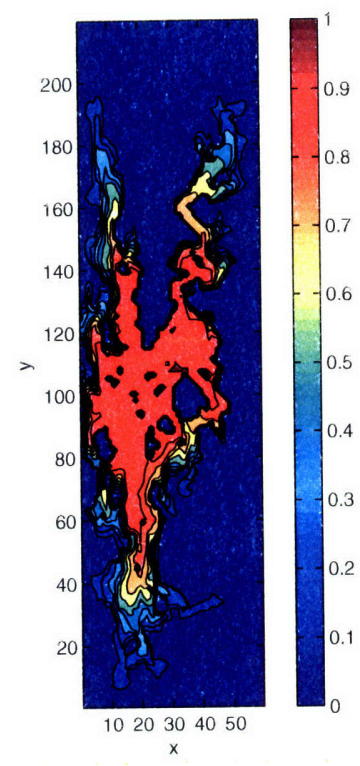

(d)

Figure 4-27: Concentration maps at breakthrough with fluxes obtained by (a) Finite volume method on the fine $60 \times 220$ grid; (b) VMS on a $6 \times 22$ grid; (b) VMS on a $30 \times 22$ grid; (b) VMS on a $30 \times 55$ grid. 

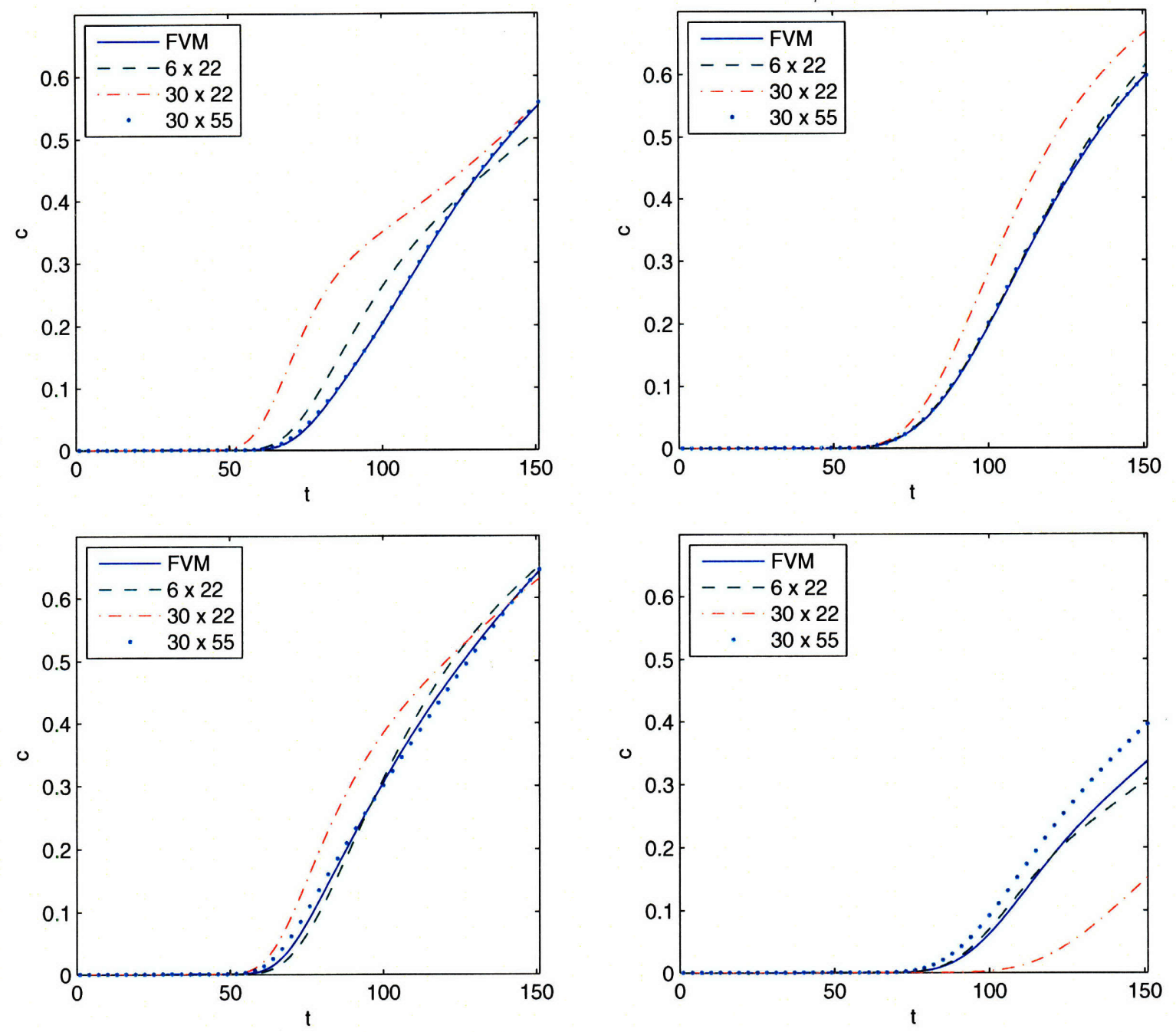

Figure 4-28: Breakthrough curves of the passive tracer at each of the production wells, computed using fine-scale fluxes from different coarse-grid discretizations. Clockwise, from top-left: Top-left well; Top-right well; Bottom-right well; Bottom-left well.

\subsubsection{All layers}

We complete our analysis of the performance of the proposed variational multiscale method by simulating the same injection-production scenario for all layers of the 10th SPE comparative solution project, which includes the two previous test cases and 83 other layers. The first 35 layers correspond to the Tarbert formation and display large, but smooth, variations in permeability. The last 50 layers correspond to the Upper Ness formation, and are characterized by a channelized permeability 
field.

Fine-scale velocity. Finite volume solutions are computed on a fine grid of $60 \times 220$ gridblocks, on which the permeability field is defined. In Figure 4-29, we plot the the velocity error of the VMSMFE solution for each layer. The multiscale solution was computed using a well length scale of $N_{l}=1 / 4$ and a $6 \times 22$ coarse grid $(10 \times 10$ upgridding). It is apparent that the errors are consistently lower for the first 35 layers, for which the permeability field is smoother.

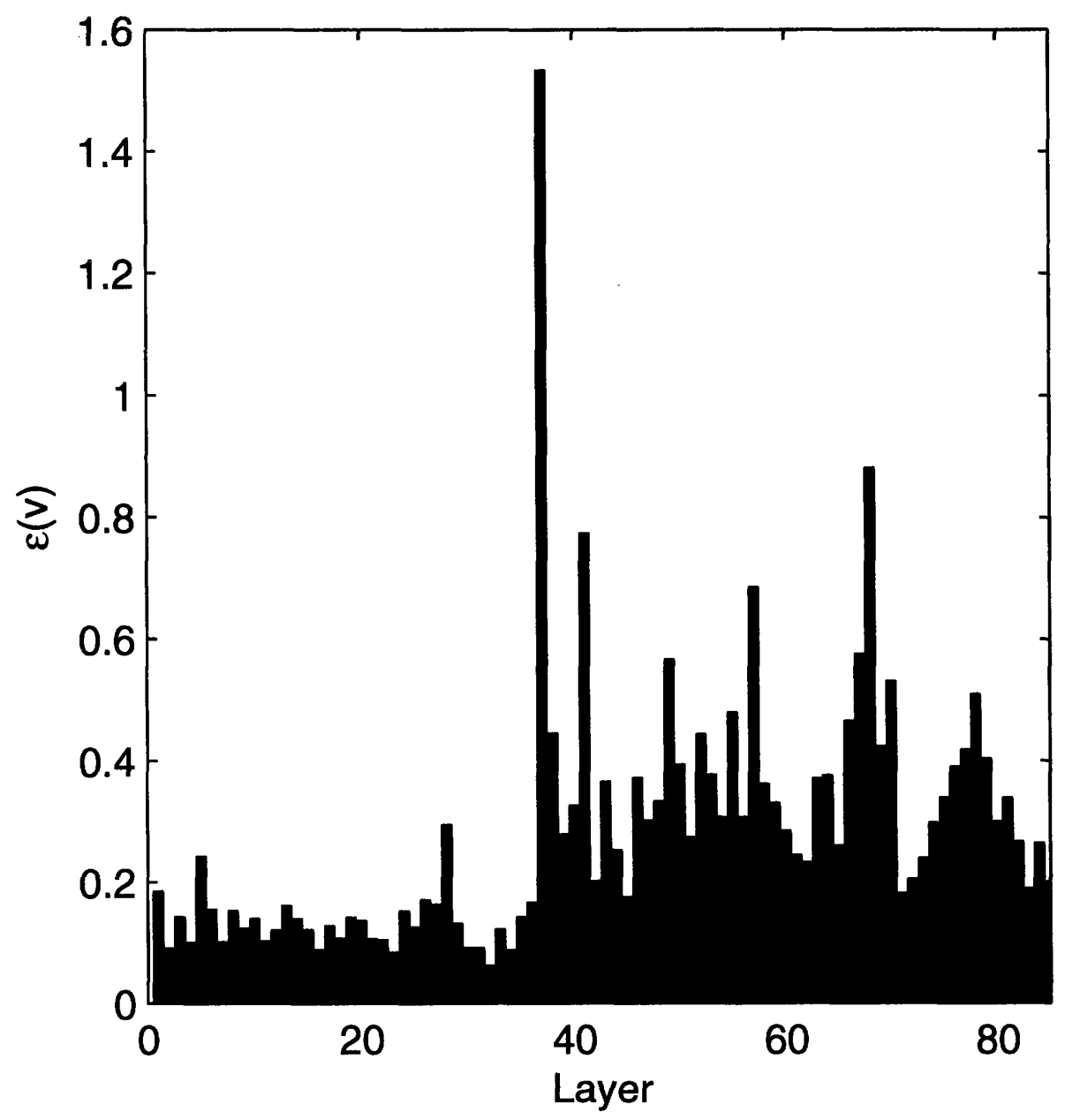

Figure 4-29: All layers of the 10th SPE comparative solution project. Velocity error.

Simulation of transport. The computed velocity error takes large values, in the range $0.3-1.0$, for the layers in the highly channelized formation. As shown in the previous subsection, however, this large velocity error does not necessarily mean that 
the VMS method fails to give accurate solutions to transport problems. To illustrate this, we simulate the transport of a passive tracer using the reconstructed fine-scale fluxes from the VMS solution on a $6 \times 22$ grid, and compare the solution to a reference finite volume solution on the fine grid. We employ a simple measure the mean concentration error (at breakthrough in the first production well), computed in the following manner:

$$
\varepsilon(c)=\frac{\left\|c-c^{\mathrm{ref}}\right\|^{2}}{\left\|c^{\mathrm{ref}}\right\|^{2}},
$$

where $c$ and $c^{\text {ref }}$ are array vectors that contain the average tracer concentration in each fine element (multiscale solution and reference finite volume solution, respectively), and $\|\cdot\|$ is the usual discrete $l^{2}$ norm.

In Figure 4-30, we plot the concentration error at breakthrough for each layer. Clearly, the error remains small, even for the challenging layers corresponding to the Upper Ness formation. The mean concentration error is $\langle\varepsilon(c)\rangle \approx 0.033$, almost an order of magnitude smaller than the average velocity error, $\langle\varepsilon(\boldsymbol{u})\rangle \approx 0.28$. These results indicate that the measure given by the velocity error is too stringent, and not entirely representative of the quality of the flow solution. 


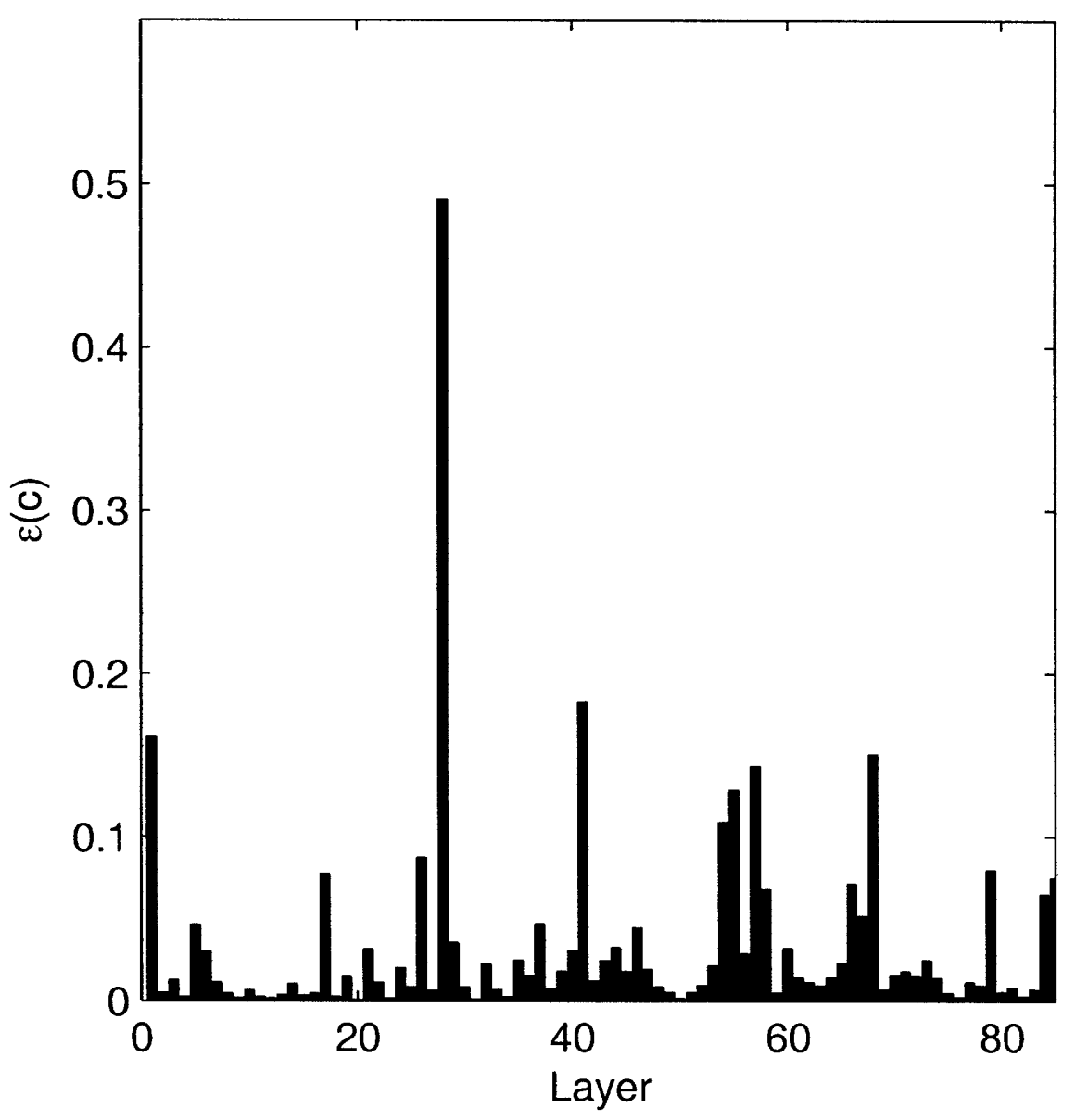

Figure 4-30: All layers of the 10th SPE comparative solution project. Concentration error.

Well pressures. In practice, well rates are dependent on the difference between the well pressure and the gridblock pressure. Therefore, it is very important that the fine-scale pressure be of high quality. Here, we measure the quality of the fine-scale pressure solution by computing a weighted pressure difference between injectors and producers. More precisely, we compute the following quantity:

$$
\Delta_{\mathrm{BHP}}:=\sum_{j=1}^{N_{w}} Q_{j} \mathrm{p}_{j},
$$

where $Q_{j}$ is the well rate (positive for injectors, negative for producers) and $\mathrm{p}_{j}$ is the well fine-scale gridblock pressure. For each layer, we compare the computed quantity 
$\Delta_{\mathrm{BHP}}$ from a fine-scale finite volume solution and the VMSMFE solution on a $6 \times$ 22 grid (100-fold upgridding) with $N_{l}=1 / 4$. This comparison is shown in Figure 4-31. For the entire range of values, $\Delta_{\mathrm{BHP}}$ from the VMS solution is remarkably accurateif the VMS solution were exact the graph would show a perfect $1: 1$ correlation. This is an essential property for the practical use of the proposed variational multiscale method in reservoir simulation.

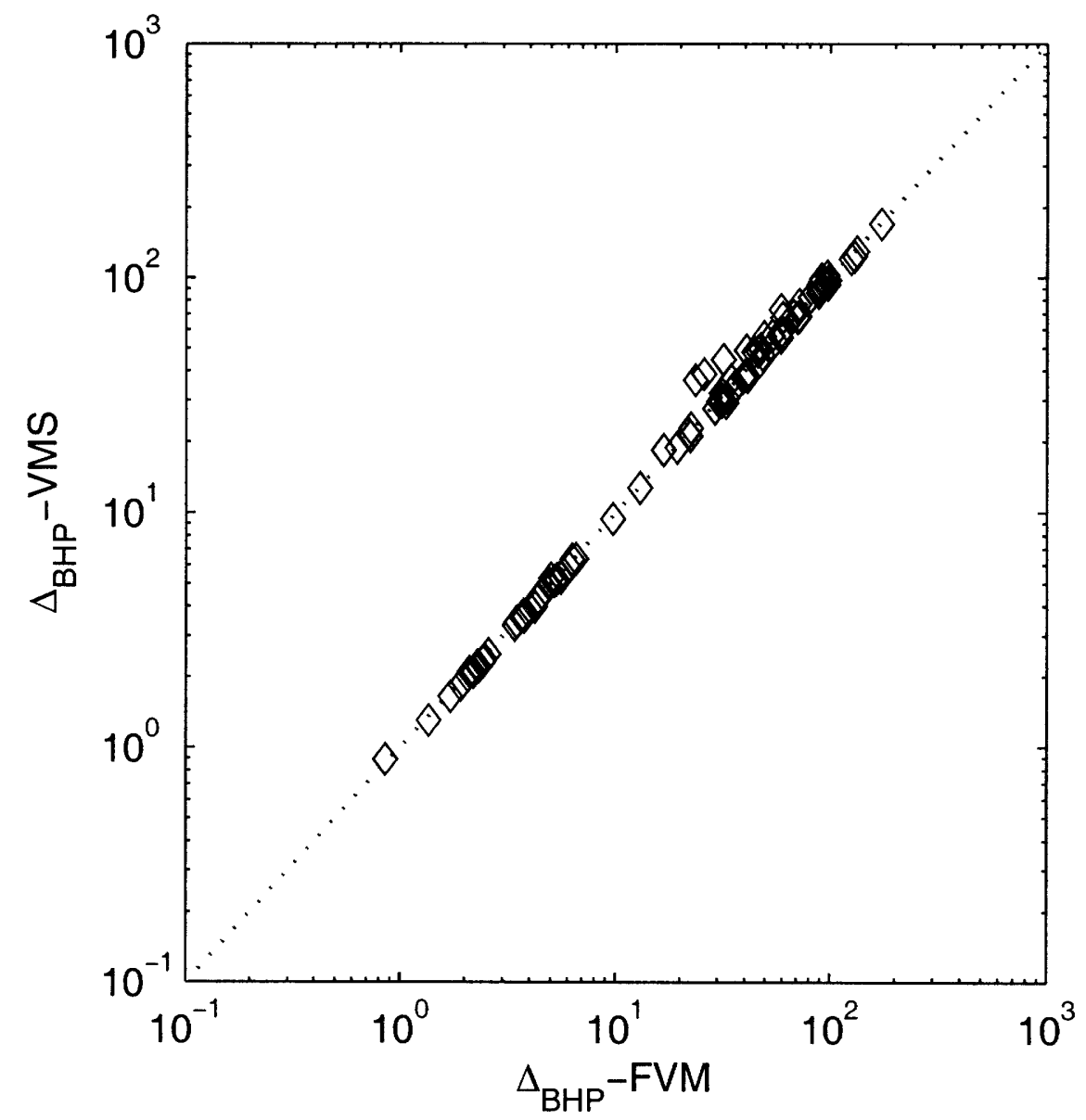

Figure 4-31: Comparison of $\Delta_{\mathrm{BHP}}$ between the reference finite volume solution and the variational multiscale solution with 100-fold upgridding for all layers of the 10th SPE comparative solution project. 


\section{Conclusions and future work}

The numerical simulations of the previous section demonstrate that the variational multiscale mixed finite element method provides highly accurate solutions to flow scenarios with challenging permeability fields and concentrated fine-scale source terms. In the spirit of many other multiscale methods, this is accomplished through the solution of a global coarse-grid problem that incorporates (rigorously, in a variational setting) the effect of the subgrid scales computed locally. The method is an extension of the numerical subgrid upscaling technique proposed in [4] and, as we show here, has a clear connection with the multiscale mixed finite element method [28]. The key ingredients of our method are:

1. A weak localization assumption of the subgrid problems that allows for subgrid communication across element interfaces.

2. A decomposition of fine-scale source terms into coarse and deviatoric components, and the definition of multiscale "well" basis functions.

The method is locally conservative, flux-continuous, and employs a low-order mixed

finite element approximation at both scales. The method, as presented here, is amenable to a number of extensions, such as:

1. Introducing a Peaceman-type well model for practical use of the method in reservoir simulation.

2. Coupling with the transport equation, using adaptivity to minimize the number of shape functions that need to be updated for the solution of the pressure 
equation, and extending it to the nonlinear regime (non-unit mobility ratio and multiphase flow).

3. Incorporating global information in the definition of the local subgrid problems, in the spirit of the coupled local-global upscaling approach $[15,18]$. 


\section{Bibliography}

[1] J. E. Aarnes. On the use of a mixed multiscale finite element method for greater flexibility and increased speed or improved accuracy in reservoir simulation. Multiscale Model. Simul., 2(3):421-439, 2004.

[2] J. E. Aarnes, V. Kippe, and K.-A. Lie. Mixed multiscale finite elements and streamline methods for reservoir simulation of large geomodels. Adv. Water Resour., 28:257-271, 2005.

[3] T. Arbogast. Numerical subgrid upscaling of two-phase flow in porous media. In Z. Chen, R. E. Ewing, and Z.-C. Shi, editors, Numerical Treatment of Multiphase Flow in Porous Media, volume 552 of Lecture Notes in Physics, pages 35-49, Berlin, 2000. Springer.

[4] T. Arbogast. Implementation of a locally conservative numerical subgrid upscaling scheme for two-phase Darcy flow. Comput. Geosci., 6(3-4):453-481, 2002.

[5] T. Arbogast. Analysis of a two-scale, locally conservative subgrid upscaling for elliptic problems. SIAM J. Numer. Anal., 42(2):576-598, 2004.

[6] T. Arbogast and K.J. Boyd. Subgrid upscaling and mixed mulitscale finite elements. SIAM J. Numer. Anal., 44(3):1150-1171, 2006.

[7] T. Arbogast and S. L. Bryant. A two-scale numerical subgrid technique for waterflood simulation. Soc. Pet. Eng. J., 7(4):446-456, December 2002.

[8] K. Aziz and A. Settari. Petroleum Reservoir Simulation. Elsevier, London, 1979. 
[9] I. Babuška. The finite element method with Lagrangian multipliers. Numer. Math., 20:179-192, 1973.

[10] I. Babuška, G. Caloz, and J.E. Osborn. Special finite element methods for a class of second order elliptic problems with rough coefficients. SIAM J. Numer. Anal., 31(4):945-981, 1994.

[11] I. Babuška and J.E. Osborn. Generalized finite element methods: their performance and relation to mixed methods. SIAM J. Numer. Anal., 20(3):510-536, 1983.

[12] F. Brezzi. On the existence, uniqueness and approximation of saddle point problems arising from Lagrange multipliers. RAIRO Anal. Numér., 8:129-151, 1974.

[13] F. Brezzi, J. Douglas, Jr., and L. D. Marini. Two families of mixed finite elements for second order elliptic problems. Numer. Math., 47:217-235, 1985.

[14] F. Brezzi and M. Fortin. Mixed and Hybrid Finite Element Methods, volume 15 of Springer Series in Computational Mathematics. Springer-Verlag, New York, 1991.

[15] Y. Chen, L. J. Durlofsky, M. Gerritsen, and X. H. Wen. A coupled local-global upscaling approach for simulating flow in highly heterogeneous formations. $A d v$. Water Resour., 26(10):1041-1060, 2003.

[16] Z. Chen and T. Y. Hou. A mixed multiscale finite element method for elliptic problems with oscillating coefficients. Math. Comp., 72(242):541-576, 2002.

[17] M. A. Christie and M. J. Blunt. Tenth SPE comparative solution project: A comparison of upscaling techniques. SPE Reserv. Eval. Eng., 4(4):308-317, 2001. url: www.spe.org/csp.

[18] L.J. Durlofsky, Y. Efendiev, and V. Ginting. An adaptive local-global multiscale finite volume element method for two-phase flow simulations. Adv. Water Resour., 30:576-588, 2007. 
[19] Y. R. Efendiev, T. Y. Hou, and X.-H. Wu. Convergence of a nonconforming multiscale finite element method. SIAM J. Numer. Anal., 37(3):888-910, 2000.

[20] T. Y. Hou and X.-H. Wu. A multiscale finite element method for elliptic problems in composite materials and porous media. J. Comput. Phys., 134:169-189, 1997.

[21] T. Y. Hou, X.-H. Wu, and Z. Cai. Convergence of a multiscale finite element method for elliptic problems with rapidly oscillating coefficients. Math. Comp., 68(227):913-943, 1999.

[22] T. J. R. Hughes. Multiscale phenomena: Green's functions, the Dirichlet-toNeumann formulation, subgrid scale models, bubbles and the origins of stabilized methods. Comput. Methods Appl. Mech. Engrg., 127:387-401, 1995.

[23] T. J. R. Hughes, G. R. Feijóo, L. Mazzei, and J.-B. Quincy. The variational multiscale method - a paradigm for computational mechanics. Comput. Methods Appl. Mech. Engrg., 166:3-24, 1998.

[24] P. Jenny, S. H. Lee, and H. A. Tchelepi. Multi-scale finite-volume method for elliptic problems in subsurface flow simulation. J. Comput. Phys., 187(1):47-67, 2003.

[25] P. Jenny, S. H. Lee, and H. A. Tchelepi. Adaptive multiscale finite-volume method for multiphase flow and transport in porous media. Multiscale Model. Simul., 3(1):50-9, 2004.

[26] R. Juanes. A variational multiscale method for the simulation of porous media flow in highly heterogeneous formations. In Computational Methods in Water Resources XVI, page 249, Copenhagen, Denmark, June 19-22 2006.

[27] R. Juanes and F.-X. Dub. A locally conservative variational multiscale method for the simulation of porous media flow with multiscale source terms. Comput. Geosci., Special Issue on Multiscale Methods for Flow and Transport in Heterogeneous Porous Media, 2(12), 2008. doi:10.1007/s10596-007-9070-x. 
[28] V. Kippe, J. E. Aarnes, and K.-A. Lie. A comparison of multiscale methods for elliptic problems in porous media flow. Comput. Geosci., Special Issue on Multiscale Methods for Flow and Transport in Heterogeneous Porous Media, 2(12), 2008. doi:10.1007/s10596-007-9074-6.

[29] S.H. Lee, C. Wolfsteiner, and H.A. Tchelepi. Multiscale finite-volume formulation for multiphase flow in porous media: black oil formulation of compressible, three phase flow with gravity. Comput. Geosci., Special Issue on Multiscale Methods for Flow and Transport in Heterogeneous Porous Media, 2(12), 2008. doi:10.1007/s10596-007-9069-3.

[30] I. Lunati and P. Jenny. Treating highly anisotropic subsurface flow with the multiscale finite-volume method. Multiscale Model. Simul., 6(1):308-318, 2007.

[31] D.W. Peaceman. Interpretation of well-block pressures in numerical reservoir simulation. In SPE Annual Technical Conference and Exhibition (SPE6893), Denver, CO, 1977.

[32] P. A. Raviart and J. M. Thomas. A mixed finite element method for second order elliptic problems. In I. Galligani and E. Magenes, editors, Mathematical Aspects of the Finite Element Method, volume 606 of Lecture Notes in Mathematics, pages 292-315, New York, 1977. Springer-Verlag.

[33] T.F. Russel and M.F. Wheeler. Finite element and finite difference methods for continuous flows in porous media. The Mathematics of Reservoir Simulation. R.E. Ewing, SIAM Philadelphia, PA, 1983.

[34] A. Weiser and M.F. Wheelert. On convergence of block-centered finite differences for elliptic problems. SIAM J. Numer. Anal., 25(2):351-375, 1988.

[35] C. Wolfsteiner, S.H. Lee, and H.A. Tchelepi. Well modeling in the multiscale finite volume method for subsurface flow simulation. Multiscale Model. Simul., 5(3):900-917, 2006. 